\title{
Les grands mouvements climatiques
}

\author{
Joseph Jacquet
}

Du point de vue du climatologue, la seconde moitié du $\mathrm{Xx}^{\mathrm{e}}$ siècle aura été marquée par un ensemble de faits remarquables :

- l'exploration de l'espace et des autres planètes qui a fait ressortir, si besoin en était, l'originalité de la nôtre en relation avec le développement de la vie;

- l'observation satellitaire pour la prise en compte de la dimension planétaire et l'exploration quantifiée de la terre à partir de l'espace par des techniques diversifiées de radiométrie, altimétrie de précision, etc...;

- le décodage des " archives " climatiques de la planète, recélées en particulier dans les dépôts de sédiments et les glaces, qui a permis de documenter, en la quantifiant, une histoire mouvementée dont les mécanismes sont encore à découvrir : ce sera la source d'informations de base de cet exposé ;

- enfin, l'explosion informatique et les progrès de l'analyse numérique, qui permettent la représentation de systèmes complexes à interactions fortes non linéaires, de règle en matière de climatologie dynamique.

Une première question s'impose : la définition du climat
planétaire sur laquelle s'appuyer pour décrire les grands
mouvements climatiques. On retiendra ici deux approches
complémentaires:
- l'une, relevant de la dynamique, présente le climat
comme résultante de la redistribution au niveau du sol
- par le jeu des échanges radiatifs et thermiques entre
l'atmosphère, les continents, les océans et la cryosphère-
de l'énergie du rayonnement solaire intercepté par la planè-
te;
- l'autre, de nature descriptive, se réfère au "temps
moyen " qui règne sur une surface donnée, identifié par la
structure des moyennes statistiques de paramètres indica-

\section{The great climatic movements}

One of the great scientific contributions of our era will no doubt remain the decoding of planetary " archives " relative to climate which have enabled successive cold and hot, humid and dry periods to be restituted over more than a million years. Quite fine quantified descriptions of the last cycles covering a period of 150000 years are now available. The explanation of these phenomena of the past - as regards both their rythm as well as their amplitude - remains an open question just as does the impact of human activities for the future: understanding (and simulation) of past fluctuations is not just a question of curiosity, they will enable questions about the future to be answered with more precision. The corresponding research effort is a vital part of the GeosphereBiosphere programme which will cover the decade 1990 and from which much is to be expected in order to have a more responsable management of planetary resources. The relatively stable climatic conditions that we have been experiencing since the historic era only account for a moment in an otherwise active timescale. Drought phenomena and desertification must be interpreted by putting them back into this context depending on factors of change relative to very diverse timescales, the long term being equal to about 20000 years (the last climatic cycle) and the short term about one century, (on the scale of anthropic factors). 
teurs de la pluviosité, de la température, de l'hygrométrie, du régime des vents, etc... qui le caractérisent. Il s'agit d'un état global d'équilibre, défini par la stationnarité des séries chronologiques correspondantes au cours de la période de référence, ce qui n'exclut pas de fortes variations autour de la moyenne, incluant des composantes saisonnières assez régulières et des fluctuations " turbulentes " d'états transitoires à échelle de temps plus fine. S'il apparait une dérive significative dans la statistique sur une longue période, on parle de changement climatique : il faut alors bien préciser les échelles de temps auxquelles on fait référence.

\section{L'exposé comporte deux parties :}

La première est un simple rappel des bases de la dynamique du climat planétaire vues à travers l'originalité de la planète Terre et de son atmosphère, le fonctionnement énergétique du système Terre-Atmosphère à partir du rayonnement solaire intercepté, de l'«effet de serre» atmosphérique et du cycle de l'eau. On évoquera ensuite la découverte du passé du climat planétaire à grande échelle, tel qu'il apparaît sous forme d'histoire conjuguée de la biosphère et du climat, par le décodage d'" archives " sédimentaires grâce aux techniques de la paléoclimatologie quantitative.

La seconde est consacrée à une présentation de l'évolution du climat planétaire au cours du temps. On a choisi pour cela 6 échelles de temps caractéristiques, du million d'années à la décennie, en mettant - dans la mesure du possible - cette évolution en relation avec les phénomènes de sécheresse et de désertification qui nous occupent aujourd'hui.

Les problèmes du futur et l'effort de recherche entrepris pour assurer la transition de la climatologie descriptive à la compréhension de la dynamique du climat, qui conditionne notamment l'évaluation de l'influence potentielle de l'homme sur le climat planétaire, concluront cet exposé.

\section{PREMIÈRE PARTIE : LES BASES DE LA CLIMATOLOGIE DYNAMIQUE ET DE LA CLIMATOLOGIE HISTORIQUE À GRANDE ÉCHELLE}

\section{1. «La Terre, une planète pas comme les autres "...}

En reprenant ce titre d'un "best-seller», on entend ici attirer l'attention sur 4 facteurs principaux d'originalité de la planète :

- la présence d'une atmosphère protectrice qui joue le rôle d'un filtre radiatif en éliminant certaines radiations nocives dans l'ultra-violet du rayonnement solaire intercepté et en piégeant par " effet de serre " une partie du rayonnement terrestre infra-rouge ;

- l'abondance de la molécule $\mathrm{H}_{2} \mathrm{O}$ dans une mince pellicule autour du globe : elle existe sous ses 3 états physiques dans l'atmosphère au voisinage du point triple de son diagramme thermodynamique. De faibles variations de pression et de température suffisent à provoquer des changements d'état avec les modifications thermodynamiques importantes qui les accompagnent (chaleur latente de vaporisation de l'eau $\cong 2500 \mathrm{~kJ} / \mathrm{kg}$, chaleur latente de fusion de la glace $\cong 300 \mathrm{~kJ} / \mathrm{kg}$, chaleur spécifique de l'eau $\cong 4$ fois celle de l'air). Le cycle de l'eau, par le biais des changements d'état qui le constituent, est ainsi un vecteur privilégié de transfert d'énergie thermique. L'atmosphère, associée au cycle de l'eau, crée en permanence les conditions nécessaires au développement de la vie dans une pellicule du globe dénommée biosphère dont le fonctionnement (échanges de matière et d'énergie, synthèses biochimiques, etc...) apparaît comme celui d'un système dynamique fortement interactif ;
— la "stabilité " du système est assurée par des cycles biogéochimiques d'éléments et de molécules $(\mathrm{C}, \mathrm{N}, \mathrm{S}, \mathrm{P}$, $\mathrm{H}_{2} \mathrm{O}$, etc...) qui conditionnent et expriment les phases du développement de la vie dans les différents compartiments de la biosphère ;

- enfin, couronnement de l'édifice : l'homme, exploitant responsable des ressources de la planète. Le mot à souligner ici est : "responsable ", car il indique clairement un objectif à atteindre et non une donnée d'entrée de jeu...

\section{Le fonctionnement énergétique du système Terre- Atmosphère conditionne la dynamique du climat}

- Le Soleil - dont on admettra ici la constance d'émission dans le temps - est la source unique d'énergie du système. L'énergie du rayonnement solaire intercepté est de l'ordre de $350 \mathrm{~W} . \mathrm{m}^{-2}$, valeur moyenne doublement lissée dans le temps (en année moyenne) et dans l'espace (rapportée à la superficie totale du globe), à comparer au flux moyen d'énergie géothermique à la surface de la planète qui est de l'ordre de $0.063 \mathrm{~W} . \mathrm{m}^{-2}$. Environ $35 \%$ du rayonnement solaire intercepté est renvoyé vers l'espace par l'albedo ou réflectivité du système Terre-Atmosphère (forte dans les régions polaires et les déserts, faible dans la forêt équatoriale et les océans), les $65 \%$ restants sont absorbés et transformés en chaleur par le système qui devient à son tour émetteur dans l'infra-rouge : il s'agit du rayonnement terrestre. 
- Le bilan radiatif et thermique du système TerreAtmosphère est globalement équilibré : la quantité d'énergie renvoyée dans l'espace par le rayonnement terrestre infrarouge est la même que celle reçue du rayonnement solaire absorbé par le système, soit environ $230 \mathrm{~W} \cdot \mathrm{m}^{-2}$. La planète se comporte thermodynamiquement comme un transformateur qui dégrade l'énergie du rayonnement solaire reçu dans la gamme de longueurs d'onde 0,35-4 $\mu \mathrm{m}$ en énergie radiative de grandes longueurs d'onde (supérieures à $4 \mu \mathrm{m}$ ) dans l'infrarouge.

- Si l'on dresse le bilan des échanges radiatifs dont l'atmosphère, d'une part, et la surface terrestre, d'autre part, sont le siège, on constate :

- pour l'atmosphère, la perte d'énergie dans l'infrarouge est supérieure à l'énergie du rayonnement solaire absorbé, d'où une tendance au refroidissement par le seul jeu des échanges radiatifs ;

- pour la surface terrestre, on observe l'inverse avec une tendance au réchauffement par le jeu des mêmes échanges.

L'équilibre thermodynamique de l'ensemble exige donc qu'il y ait en permanence des transferts d'énergie de la surface terrestre vers l'atmosphère par processus nonradiatifs, c'est-à-dire des transferts turbulents de chaleur de l'ordre de $95 \mathrm{~W} . \mathrm{m}^{-2}$ en moyenne, dont $80 \%$ s'opèrent sous forme de chaleur latente par évaporation de l'eau et $20 \%$ sous forme de chaleur sensible par conduction et convection.

- Ces 95 W.m-2 $\mathrm{m}^{-2}$ sont moteurs des circulations atmosphérique et océanique qui assurent la redistribution de l'énergie excédentaire reçue dans les régions intertropicales vers les régions polaires, d'oủ résulte le climat planétaire. Le fonctionnement énergétique du système Terre-Atmosphère est celui d'une machine thermique dont la source chaude est la zone intertropicale et la source froide les zones polaires, les fluides de travail l'air humide et l'eau océanique, et le mouvement représenté par le régime des vents et des courants à circulation cyclonique (et non méridienne) du fait de la rotation de la terre.

A l'issue de ces brefs rappels, deux conclusions s'imposent :

- le couplage des dynamiques atmosphérique et océanique, associées au cycle de l'eau, est le facteur essentiel de la distribution de la chaleur et, par conséquent, des climats à la surface du globe : ce couplage devra obligatoirement être pris en compte par tous les modèles représentatifs de climatologie dynamique ;

- l'atmosphère est chauffèe "par le bas", expliquant ainsi le gradient vertical négatif de température de la troposphère, et la valeur de $95 \mathrm{~W} \cdot \mathrm{m}^{-2}$ des transferts de chaleur sensible et latente constitue une norme de référence pour apprécier l'impact climatique possible des rejets de chaleur consécutifs à l'utilisation des ressources planétaires par l'homme.

On ne peut micux faire que de citer ici ce qu'écrivait Sadi CARNOT en 1824 dans ses "Réflexions sur la puissance motrice du feu " :
... «C'est à la chaleur que doivent être attribués les grands mouvements qui frappent nos regards sur la terre; c'est à elle que sont dues les agitations de l'atmosphère, l'ascension des nuages, la chute des pluies et des autres météores, les courants d'eau qui sillonnent la surface du globe et dont l'homme est parvenu à employer pour son usage une faible partie "...

\section{Le fonctionnement du "filtre» atmosphérique et ses effets protecteurs}

- La fonction «filtre " de l'atmosphère apparaît à travers 2 types de comparaison des rayonnements reçus et émis par le système Terre-Atmosphère: d'une part, quand on compare la répartition spectrale du rayonnement solaire telle qu'elle se présente à la limite supérieure de l'atmosphère à celle de ce même rayonnement au niveau de la mer (fig. I, page suivante), et d'autre part dans l'analyse du spectre du rayonnement terrestre après la traversée de l'atmosphère (fig. 2).

- Deux effets protecteurs se révèlent ainsi :

- l'absorption d'une grande partie de l'ultraviolet solaire par la couche d'ozone strastosphérique dont le rôle est essentiel pour assurer la non-nocivité biologique du rayonnement reçu à la surface du globe ;

- l'absorption du rayonnement terrestre infrarouge dans des bandes spectrales correspondant aux propriétés radiatives de gaz dits " actifs", qui existent à l'état de trace dans l'atmosphère soit comme constituants minoritaires $\left(\mathrm{H}_{2} \mathrm{O}\right.$, $\mathrm{CO}_{2}, \mathrm{O}_{3}, \mathrm{~N}_{2} \mathrm{O}, \mathrm{CH}_{4}$ ), soit en tant que polluants introduits par l'homme (cas des fréons, composés chlorofluoro-carbonés, par exemple). L'action de ces gaz se manifeste sous forme de "trous " dans la région infrarouge du spectre du rayonnement solaire reçu à la surface du globe et surtout dans le spectre d'émission infrarouge de la terre mesuré à partir de l'espace. Nous nous intéresserons particulièrement ici à ce second effet protecteur de l'atmosphère car il est à la base de l'" effet de serre", propriété fondamentale de l'atmosphère dans sa relation au climat.

- La dénomination "effet de serre» a été choisie par analogie avec le fonctionnement des serres horticoles où l'on protège des cultures fragiles au moyen de vitres qui laissent passer l'énergie du rayonnement solaire dans le visible mais sont "opaques" à la fuite directe vers l'extérieur de l'énergie du rayonnement infrarouge émis à l'intérieur de l'enceinte. Le mécanisme de l' « effet de serre " atmosphérique réside en ce que les molécules de gaz qui ont absorbé des fractions de rayonnement terrestre émettent à leur tour dans les mêmes bandes spectrales et renvoient vers la surface du globe une partie de l'énergie piégée, ce qui se traduit par une diminution de l'énergie radiative perdue par cette surface.

- Les bandes d'absorption dans l'infrarouge sont bien différenciées et spécifiques aux différents gaz « actifs » (sauf en ce qui concerne $\mathrm{H}_{2} \mathrm{O}$ dont le spectre d'absorption infrarouge est très étendu) : $\mathrm{CH}_{4}, \mathrm{~N}_{2} \mathrm{O}, \mathrm{O}_{3}$ troposphérique sont surtout absorbants dans la bande $7-12 \mu \mathrm{m}$ tandis que $\mathrm{H}_{2} \mathrm{O}$ 


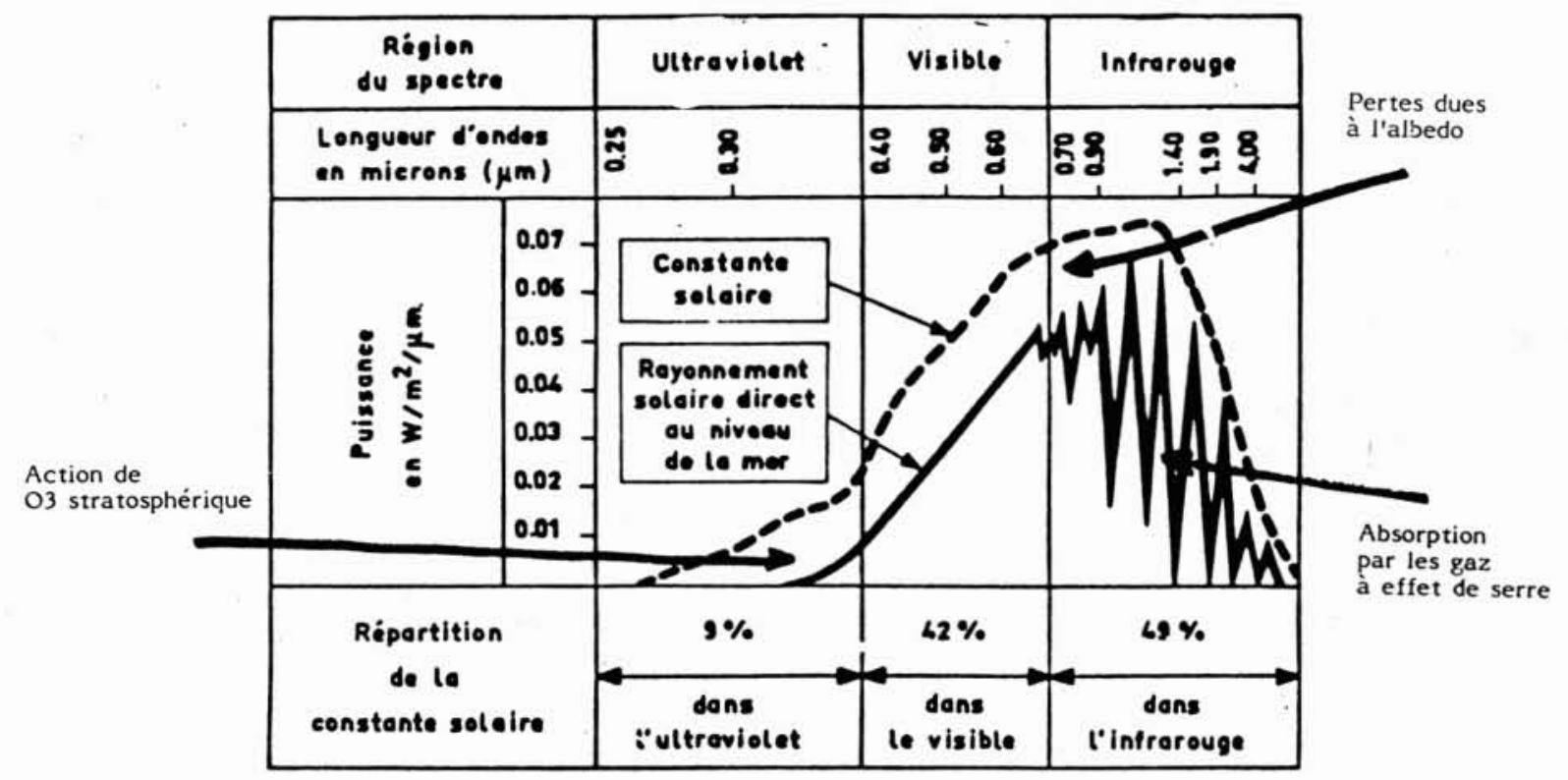

1. Modification de la répartition spectrale du rayonnement solaire dans la traversée de l'atmosphère. Le spectre solaire hors de l'atmosphère est assez proche de celui de l'émission d'un corps noir à $5900 \mathrm{~K}$ et son énergie représente la constante solaire. Le graphique met en évidence l'action de l'ozone stratosphérique dans la région de l'ultraviolet, l'absorption dans l'infrarouge par les gaz " radiativement actifs » de l'atmosphère et les pertes de rayonnement par réflexion dues à l'albedo du système Terre-Atmosphère.

2. Spectre d'émission infrarouge de la terre mesuré de l'espace par le Satellite Nimbus 4. Les courbes régulières représentent les spectres d'émission du corps noir à différentes températures: le spectre d'émission de la terre correspond à celui d'un corps noir à $288 \mathrm{~K}\left(+15^{\circ} \mathrm{C}\right)$. Les 2 " trous" principaux du spectre correspondent aux bandes d'absorption de $\mathrm{CO}_{2}$ et de $\mathrm{O}_{3}$, celles de $\mathrm{H}_{2} \mathrm{O}$ sont très étalées.

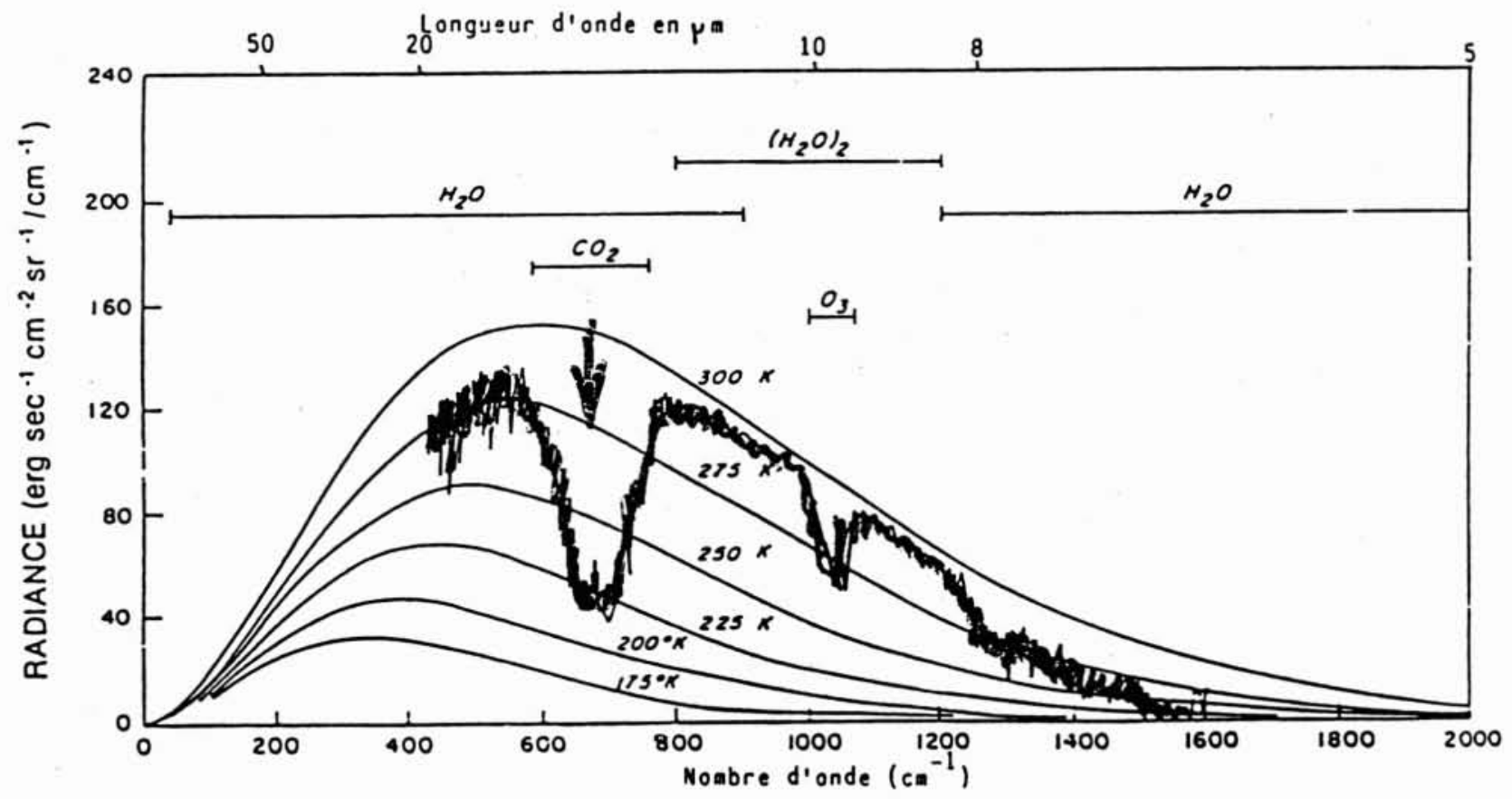


Tableau 1. Repères d'évolution des concentrations atmosphérique de gaz à effet de serre.

\begin{tabular}{|c|c|c|c|c|}
\hline \multirow{2}{*}{$\begin{array}{l}\text { Gaz à effet } \\
\text { de serre }\end{array}$} & \multicolumn{2}{|c|}{$\begin{array}{l}\text { Concentration volumique } \\
\text { dans la troposphère }\end{array}$} & \multirow{2}{*}{$\begin{array}{c}\text { Taux de croissance } \\
\text { annuel moyen } \\
\text { des concentrations } \\
(\%)\end{array}$} & \multirow{2}{*}{$\begin{array}{l}\text { Temps de résidence } \\
\text { dans la troposphère } \\
\text { (années) }\end{array}$} \\
\hline & époque préindustrielle & en 1980 & & \\
\hline $\begin{array}{l}\mathrm{CO}_{2} \\
\mathrm{CH}_{4} \\
\mathrm{~N}_{2} \mathrm{O} \\
\text { Fréons : } \\
\mathrm{CFCl}_{3} \\
\left(\mathrm{~F}_{11}\right) \\
\mathrm{CF}_{2} \mathrm{Cl}_{2} \\
\left(\mathrm{~F}_{12}\right) \\
\mathrm{CF}_{3} \mathrm{Cl} \\
\left(\mathrm{F}_{13}\right. \\
\mathrm{CF}_{4} \\
\left(\mathrm{~F}_{4} 14\right) \\
\mathrm{CHClF}_{2} \\
\left(\mathrm{~F}_{2} 2\right) \\
\mathrm{CH}_{3} \mathrm{CCl}_{2}\end{array}$ & $\begin{array}{c}275 \\
\text { (ppmv) } \\
700 \\
\text { (ppbv) } \\
280 \\
\text { (ppbv) }\end{array}$ & $\begin{array}{c}340 \\
\text { (ppmv) } \\
1630 \\
\text { (ppbv) } \\
310 \\
\text { (ppbv) } \\
\\
0,18 \\
\text { (ppbv) } \\
0,30 \\
\text { (ppbv) } \\
0,007 \\
\text { (ppbv) } \\
0,07 \\
\text { (ppbv) } \\
0,06 \\
\text { (ppbv) } \\
0,14 \\
\text { (ppbv) }\end{array}$ & $\begin{array}{l}0,5 \\
1,5 \\
0,4\end{array}$ & $\begin{array}{c}80 \\
170 \\
400 \\
>500 \\
20 \\
8\end{array}$ \\
\hline
\end{tabular}

et $\mathrm{CO}_{2}$ le sont principalement dans la gamme des longueurs d'onde supérieures à $12 \mu \mathrm{m}$. Ces spécificités expliquent la sensibilité de l'effet de serre aux variations de concentrations atmosphériques des gaz agents.

- La vapeur d'eau atmosphérique absorbe environ $27 \%$ de l'énergie du rayonnement terrestre, répartis dans de très larges bandes spectrales $\left(\mathrm{CO}_{2}: 16 \%\right)$. Comme il n'y a pas lieu de penser que se produise une évolution significative de la quantité d'eau atmosphérique globale, l'effet de serre engendré ne devrait pas se modifier. Par contre, on constate depuis le début de l'ère industrielle une augmentation des concentrations atmosphériques des principaux autres gaz à effet de serre, avec des taux de croissance souvent spectaculaires, sous l'effet des activités humaines (cf. tableau l). L'action combinée de ces gaz est évaluée actuellement à $60 \%$ de celle du seul $\mathrm{CO}_{2}$ et elle pourrait l'égaler dans les décennies à venir. On se trouve donc confronté à un accroissement inéluctable de l'effet de serre atmosphérique à l'échelle du prochain siècle. Dans l'état actuel des connaissances, il n'est pas possible d'évaluer avec fiabilité la réponse climatique du système planétaire à une modification donnée d'effet de serre, compte tenu de la complexité des processus et des boucles de rétroaction en jeu.

- On notera dans le Tableau $l$ que les temps de résidence de certains gaz à effet de serre (oxyde nitreux, fréons) dans la troposphère dépassent quelquefois le siècle : un effet retard considérable va donc affecter l'efficacité de mesures volontaristes qui seraient prises pour réduire et inverser la tendance...
- Il est remarquable que la fonction de protection thermique de la planète soit régie par des constituants minoritaires de l'atmosphère dont les concentrations se mesurent en parties par million et par milliard et qui représentent moins de $5.10^{-5}$ de son volume, alors que les 3 constituants majoritaires (oxygène, azote, argon : $99,96 \%$ de l'atmosphère en volume) sont pratiquement transparents au rayonnement solaire dans les parties visible et infrarouge de son spectre.

- Quelques points de repères thermiques permettront ici d'apprécier l'efficacité de l'effet de serre atmosphérique:

La température des espaces sidéraux est de $-270^{\circ} \mathrm{C}$. Sans atmosphère et sans apport d'énergie solaire, la température de la Terre résultant de son flux de chaleur propre (essentiellement dû à la radioactivité des roches) serait de $-243^{\circ} \mathrm{C}$. Sans atmosphère mais avec l'énergie solaire interceptée et absorbée en tenant compte de l'albedo, la température d'équilibre radiatif au sol serait de $-18^{\circ} \mathrm{C}$ (ce qui correspond à la température mesurée à l'extérieur du système Terre-Atmosphère en équilibre thermique avec l'espace). Avec l'atmosphère et son effet de serre, la température d'émission du spectre infrarouge du rayonnement terrestre est $+15^{\circ} \mathrm{C}(288 \mathrm{~K})$ : on peut ainsi évaluer l'effet de serre à $+33^{\circ} \mathrm{C}$. Enfin si l'on supprimait la composante $\mathrm{CO}_{2}(350 \mathrm{ppmv})$ de l'atmosphère, la température de la basse atmosphère diminuerait de $5^{\circ} \mathrm{C}$ dans les régions polaires et de $1{ }^{\circ} \mathrm{C}$ dans les régions équatoriales, accentuant ainsi les disparités énergétiques internes de la planète... Il s'agit là bien évidemment d'ordres de grandeur soumis à révisions avec le progrès des connaissances et de la fiabilité des modèles de simulation. 
On retiendra en conclusion l'impact attendu des activités humaines sur l'effet de serre atmosphérique et les incertitudes qui entourent la réponse du système climatique à cette perturbation. L'homme sera au cœur du débat sur le climat du futur qui s'insère dans une histoire mouvementée dont on déplie progressivement les traits.

\section{L'histoire du climat planétaire à grande échelle révélée par la paléoclimatologie quantitative}

- La révélation de cette histoire - conjuguée à celle de la biosphère -, grâce au décodage d'" archives " climatiques contenues dans les dépôts sédimentaires de la planète, a constitué au cours des décennies passées une aventure scientifique et humaine extraordinaire à laquelle les chercheurs et laboratoires français ont apporté une contribution de premier plan. Il suffit de citer ici Claude LoRIus, Jacques LABEYRIE, Jean-Claude DuPLESSY, animateurs des équipes du Laboratoire de glaciologie et de géophysique de l'environnement à Grenoble et du Laboratoire CNRSCEA des faibles radioactivités à Gif-sur-Yvette, et toutes les autres équipes que leur dynamisme a mobilisées pour concourir à la mise au point d'une paléoclimatologie quantitative, découvrant sans cesse de nouveaux indices à analyser au moyen de techniques remarquables par leur complémentarité et dont la fiabilité est reconnue au niveau international. Une description des climats du passé, dont la précision s'affirme au fil du temps, a vu le jour: la question de l'explication des grands mouvements climatiques identifiés reste ouverte.

- Les témoins et indicateurs climatiques du passé sont renfermés dans des séquences de dépôts sédimentaires marins, continentaux et glaciaires ou dans des matériaux fossiles. Leur décodage comporte invariablement deux séries d'opérations : la datation du dépôt lui-même et l'extraction du message de l'indicateur, considéré soit isolément soit rapporté à un assemblage d'indices, avec « in fine " son interprétation en termes de paramètres climatiques. Ces témoins se caractérisent par leur très grande variété : bulles d'air piégées depuis des dizaines de milliers d'années dans la glace, dépôts de poussières, pollens fossiles, variation de composition isotopique de l'oxygène, débris végétaux et animaux, dépôts coralliens, vestiges archéologiques, produits d'érosion, etc... : leur traitement a donc nécessité la mise au point de techniques très diversifiées.

- Les techniques de la paléoclimatologie quantitative relèvent de nombreuses disciplines que l'on peut regrouper en 3 grandes catégories : les techniques de datation, les mesures d'abondance isotropique ${ }^{18} \mathrm{O} /{ }^{16} \mathrm{O}$ et des méthodes d'analyses ultrafines appliquées à l'étude d'indicateurs microscopiques extraits des dépôts étudiés.

La datation est essentiellement basée sur la décroissance de radioactivité de certains éléments en fonction du temps en utilisant notamment des isotopes à désintégration radioactive lente. Ainsi le ${ }^{14} \mathrm{C}$, fixé dans des débris végétaux et animaux, dont la période de décroissance radioactive est de 5568 ans, permet de faire des datations jusque vers 40000 ans et, grâce aux progrès de la superspectrométrie de masse, on pense atteindre 75000 ans. La méthode Uranium-Thorium, basée sur la croissance du Thorium 230 (période : 75200 ans) dans l'Uranium 234 est utilisée jusque vers 300000 ans. La technique PotassiumArgon a permis la datation des inversions magnétiques sur des coulées de laves continentales jusqu'à plus de 1 million d'années. On mentionnera aussi la thermoluminescence, méthode de datation intéressante pour les matériaux soumis à l'épreuve du feu (céramiques, pierres de foyer, etc...). On dispose ainsi d'un éventail de techniques de datation couvrant plus d'un million d'années. Toutes ces techniques sont fiables, mais leur mise en œuvre requiert des précautions, même dans les laboratoires spécialisés. La datation par ${ }^{14} \mathrm{C}$ est la plus précise (sa précision brute est de l'ordre de 200 ans vers 25000 ans), mais ses résultats sont entâchés d'une erreur mal connue et qui peut être très importante pour passer de l'âge ${ }^{14} \mathrm{C}$ mesuré à l'âge calendaire réel : cette erreur tient notamment au fait que la concentration de ${ }^{14} \mathrm{C}$ dans l'atmosphère et le rapport ${ }^{14} \mathrm{C} /{ }^{12} \mathrm{C}$ dans les végétaux varient selon l'époque considérée sans que l'on connaisse ces variations. Le calcul de l'âge ${ }^{14} \mathrm{C}$ se fait en supposant que ce rapport est constant : on a pu comparer âges ${ }^{14} \mathrm{C}$ et âges réels tout au long des 8000 dernières années grâce à une échelle dendrochronologique basée sur une suite continue de troncs d'arbres, mais audelà de 8000 ans l'amplitude de l'erreur introduite est mal connue. C'est pourquoi l'on continue de publier les âges ${ }^{14} \mathrm{C}$ non rectifiés en les faisant suivre de la mention BP (Before Present). Si l'on ajoute à l'âge la mention AD (Anno Domini) ou BC (Before Christ), il s'agit d'âges calendaires après correction de dendrochronologie sur l'âge ${ }^{14} \mathrm{C}$. Les autres techniques de datation fournissent des dates absolues, les décroissances radioactives des éléments utilisés étant indépendantes de tout effet géophysique.

Le second groupe de techniques paléoclimatologiques se rapporte aux mesures d'abondance isotopique ${ }^{18} \mathrm{O} /{ }^{16} \mathrm{O}$. La mesure de ce rapport dans l'oxygène de la glace et dans celui des carbonates des coquilles de micro-escargots du plancton marin (foraminifères) est un extraordinaire indicateur des conditions climatiques de formation de cette glace ou de ces coquillages. On mesure en fait la variation de ce rapport - notée $\delta{ }^{18} \mathrm{O} /{ }^{16} \mathrm{O}$ - par rapport à une valeur de référence qui est le rapport ${ }^{18} \mathrm{O} /{ }^{16} \mathrm{O}$ remarquablement constant de l'eau de mer actuelle. Dans le cas de la glace, plus la température de formation de la neige qui va constituer le dépôt glaciaire est basse et plus le « déplacement isotopique " $\left(\delta^{18} \mathrm{O} /{ }^{16} \mathrm{O}\right)$ est grand : ce déplacement constitue donc un véritable "thermomètre fossile " et l'on peut ainsi pratiquement reconstituer les températures du passé en mesurant ce $\delta$ tout au long d'une carotte de neige ou de glace. Dans le cas des foraminifères, l'oxygène retiré de l'eau de mer pendant la vie de l'organisme pour constituer le carbonate de sa coquille suit la teneur isotopique de l'eau de mer, laquelle est fonction du volume des glaces accumulées sur les calottes polaires : au moment du maximum glaciaire, l'eau de mer est devenue plus « lourde " en ${ }^{18} \mathrm{O}$ par rapport à l'état actuel et on peut admettre qu'il y a proportionnalité entre le volume des glaces stockées et 
les déplacements isotopiques correspondants mesurés dans les coquilles des foraminifères. On dispose ainsi d'un indicateur de l'évolution des calottes polaires et du niveau marin qui est sous la dépendance directe des quantités de glaces stockées dans ces calottes...

La $3^{\mathrm{e}}$ catégorie des techniques paléoclimatologiques fait appel à un ensemble diversifié de techniques pour reconstituer à une date donnée l'environnement planétaire: la composition de l'atmosphère en espèces minoritaires $\left(\mathrm{CO}_{2}\right.$, $\mathrm{N}_{2} \mathrm{O}$ ) et en aérosols, le paysage à partir de l'identification des pollens fossiles et la description des associations végétales en relation avec le climat. On accède ainsi à l'évaluation de conditions thermiques en milieu marin et hydrométéorologiques (pluviosité, température) en milieu continental, d'optimum de développement ou de survie d'espèces animales ou végétales en fonction de l'évolution des caractéristiques de leur biotope, et cela en incluant l'essor et le déclin des civilisations révélés par l'archéologie en rapport avec des événements climatiques.

- Les "dépôts d'archives » explorés sont en premier lieu constitués par des « carottes " prélevées dans des sédiments marins (certaines couvrent 2 millions d'années, c'est-à-dire la totalité de l'ère quaternaire), dans les calottes glaciaires (sur le site de VOSTOK dans l'Antarctique, une carotte de $2000 \mathrm{~m}$ représente 160000 ans de dépôts de glace) et dans les sédiments continentaux où l'on a pu reconstituer des séquences polliniques sur 150000 ans. Il faut ajouter à cela l'exploration des moraines jalonnant le retrait des glaciers et calottes glaciaires, des rivages fossiles marins et lacustres, et de certains sites archéologiques.

- Les opérations de "décodage " aboutissent enfin à une batterie de paramètres climatiques quantifiés sous forme de séquences chronologiques de températures de l'air, de températures de surface et de fond de la mer, de volumes de glace et d'extensions géographiques des calottes glaciaires, de niveaux marins, de teneurs en $\mathrm{CO}_{2}$ atmosphérique, etc... Des informations plus fragmentaires concernent les paramètres hydrologiques tels que la pluviosité, l'abondance des cours d'eau ou encore le régime des vents et la circulation océanique.

- La paléoclimatologie peut afficher une extraordinaire moisson de résultats qui continue de s'enrichir au fil du temps. Elle a permis de définir et de quantifier l'évolution du climat planétaire sur plus d'un million d'années avec ses incidences sur la biosphère en reconstituant des "paysages " caractéristiques. Des cycles d'évolution ont été mis en évidence, sans faire apparaître pour autant des périodicités définies, entrecoupés qu'ils sont de stagnations et d'accélérations sans organisation apparente.

- La recherche des causes d'évolution et des mécanismes explicatifs de ces grands mouvements climatiques est ouverte dans 3 directions principales:

- la voie astronomique, avec notamment les variations de paramètres orbitaux de la terre qui affectent directement la quantité d'énergie du rayonnement solaire reçue aux différentes latitudes, ou encore avec l'hypothèse d'une variation de la constante solaire, dont on pense qu'une variation de $4 \%$ entraînerait sur l'ensemble de la surface terrestre une différence de température du même ordre qu'entre la période chaude actuelle et le dernier maximum glaciaire ;

- la voie géophysique, faisant appel aux anomalies de température et de circulation océaniques, phénomènes dont l'origine est à découvrir, ou à des activités volcaniques intenses qui injectent jusque dans la stratosphère d'importantes quantités de gaz et de poussières qui modifient la fonction " filtre " de l'atmosphère : on retrouve des traces de périodes de volcanisme intense dans les " archives " glaciaires notamment ;

- la voie biologique, avec des modifications des phases atmosphériques de cycles biogéochimiques qui peuvent affecter l'effet de serre. C'est dans cette dernière voie que l'action potentielle de l'homme devra être prise en compte.

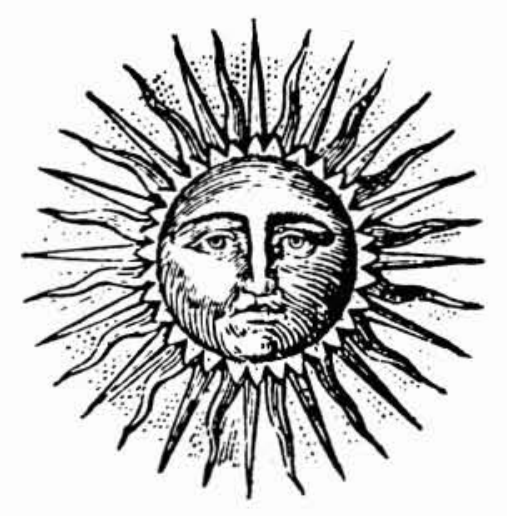




\section{$2^{\mathrm{E}}$ PARTIE : \\ PRÉSENTATION DE L'ÉVOLUTION DU CLIMAT PLANÉTAIRE À DIVERSES ÉCHELLES DE TEMPS}

Le fil directeur de cette présentation est celui des échelles temporelles régressives du million d'années à la décennie qui interfèrent dans cette évolution sans que l'on en saisisse aujourd'hui les mécanismes. On se limitera donc à une description des types d'évolution de phénomènes ou d'in- dicateurs climatiques identifiés à chacune des 6 échelles choisies, description qui devient évidemment plus précise et documentée au fur et à mesure que l'on se rapproche du présent qui sert de point de référence commun.

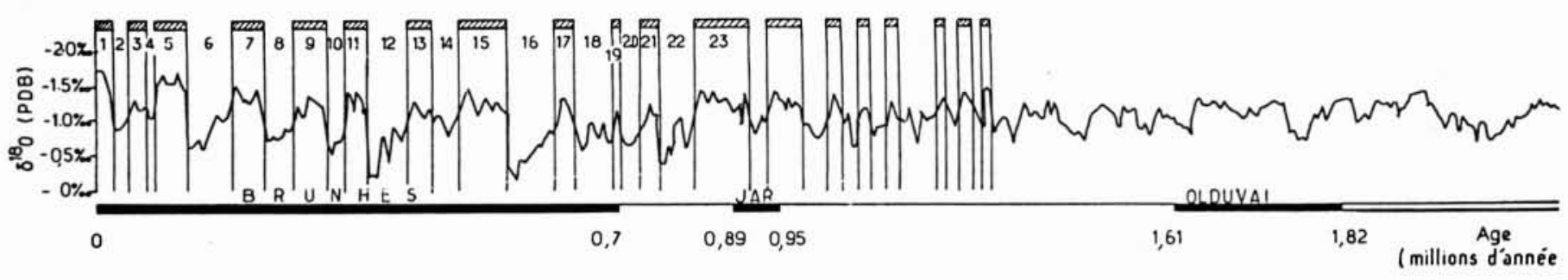

3. Suite des glaciations et des périodes chaudes du quaternaire. Les ordonnées sont graduées en \% de la variation du rapport ${ }^{18} \mathrm{O} /{ }^{16} \mathrm{O}$ dans les coquilles de foraminifères d'une carotte prélevée dans le Pacifique. Ces variations sont proportionnelles à la quantité de glaces stockées dans les calottes continentales et donc à la baisse de niveau de la mer par rapport au niveau actuel. On voit apparaittre une vingtaine de périodes glaciaires suivies d'une période chaude et donc autant de cycles climatiques au cours du quaternaire qui commence au début de la période magnétique "directe " d'Olduwaï (d'après J. LABEYRIE, 1984).

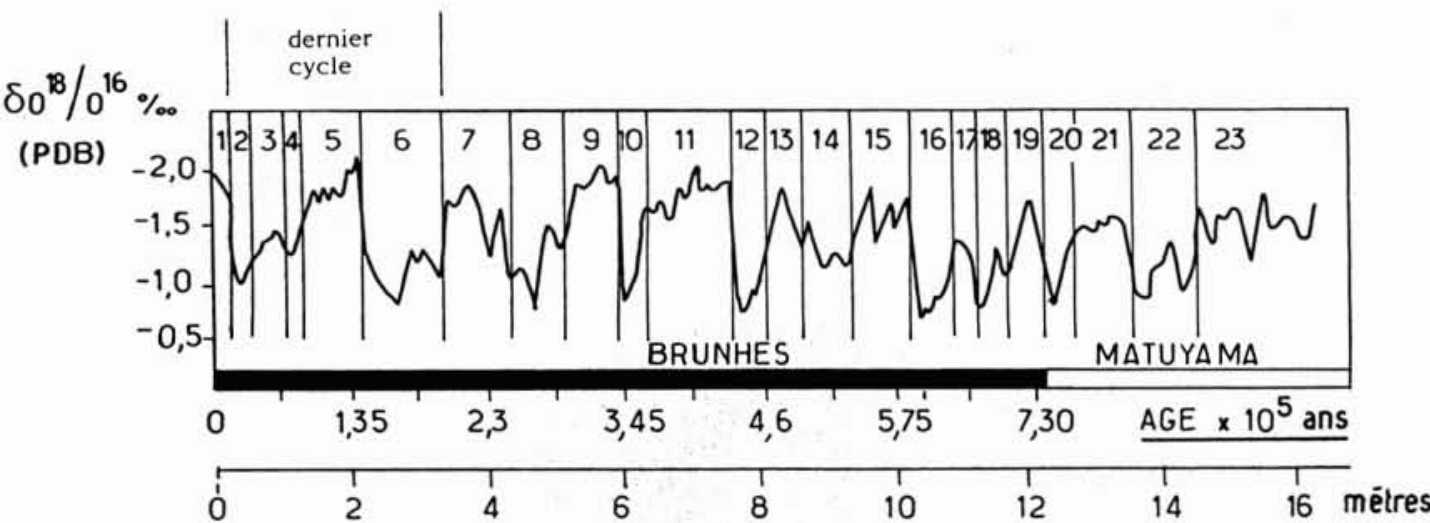

4. Les 8 derniers cycles du quaternaire. Cette courbe couvre les derniers 900000 ans avec 8 cycles climatiques. Elle provient d'une autre carotte prélevée dans le Pacifique équatorial. Les maxima de la courbe figurent les climats chauds et les minima les climats froids. Les numéros sont ceux des "stades " d'EMI. LIANI (1955): impairs pour les stades chauds, pairs pour les stades froids. Le dernier cycle climatique comporte 4 stades (de la frontière 6-5 à celle qui sépare les stades 2 et 1): les cycles précédents comportent 2 stades chacun. Les polarités magnétiques: Brunhes (normale) et Matuyama (inverse) sont indiquées en dessous. Ces 8 cycles sont révélés sur une hauteur de 16 mètres de la carotte analysée (d'après J. LABEYRIE, 1984). 


\section{Echelle du million d'années : la "turbulence " des successions de périodes chaudes et froides au cours du quaternaire}

- En prenant le dernier million d'années comme référenciel temporel, on constate une "agitation " climatique considérable: plus d'une vingtaine de cycles (périodes glaciaires suivies de périodes chaudes) ont été identifiés au cours du quaternaire qui, d'après les conventions actuelles, débute à -1.82 millions d'années (fig. 3). Les 8 derniers cycles couvrent 900000 ans (fig. 4). Pendant les périodes froides des 6 derniers cycles (600 000 ans), le Sahara était sans végétation : on observe en effet une recrudescence des dépôts de poussières silicieuses d'origine éolienne à chacune des périodes froides de ces cycles dans des carottes de sédiments prélevés dans l'Atlantique au large du Sahara. En période chaude, la végétation fixe le sol et l'on ne trouve plus ce type de matériaux dans les sédiments correspondants.

- Pour tenter d'expliquer ces séquences de cycles, on a remis en honneur la théorie astronomique de MILANKo. VITCH qui, de 1930 à 1941, a calculé, sur 600000 ans et pour 8 latitudes, les variations de l'ensoleillement, donc des apports d'énergie du rayonnement solaire, en fonction des variations de 3 paramètres orbitaux de la terre : précession des équinoxes (l'axe des pôles décrit un cône autour de la perpendiculaire au plan de l'écliptique, avec une période de 21000 ans), obliquité (l'angle de l'axe polaire avec la perpendiculaire au plan de l'écliptique varie entre 22 et $24^{\circ} 5$, avec une période approximative de 40000 ans) et excentricité de l'orbite (qui varie entre 0 et 0,07 avec une période de l'ordre de 100000 ans. fig. 5). En combinant les effets de ces 3 phénomènes, on peut aussi bien calculer les insolations du passé que celles de l'avenir (fig.6) et le professeur André BERGER, de l'Université de Louvain, a récemment étendu à 1 million d'années les calculs de MiLANKOVITCH. On observe une bonne concordance entre les variations d'insolation calculées et les 10 ou 12 derniers cycles climatiques du quaternaire, avec un décalage d'environ 2000 ans. On constate aussi que ce sont les successions d'étés froids - et non celles d'hivers froids - qui sont les facteurs prépondérants d'apparition des périodes glaciaires. L'extrapolation du calcul dans le futur indique que les mécanismes astronomiques en question devraient induire une baisse de température dans les prochains siècles, avec un léger accroissement des glaces polaires au cours du prochain millénaire, en accentuant l'aridité des régions sèches. Le prochain maximum glaciaire, abaissant de 50 mètres le niveau marin, ne devrait pas intervenir avant 50000 ans... Mais il faut souligner aussitôt les insuffisances de la théorie astronomique pour expliquer, par exemple, la constitution des calottes de glace qui dépend non seulement de la température mais aussi du cycle de l'eau qui n'est pas pris en compte ici, de même que la fréquence, l'emplacement, le déplacement et l'intensité des structures atmosphériques régissant le phénomène.

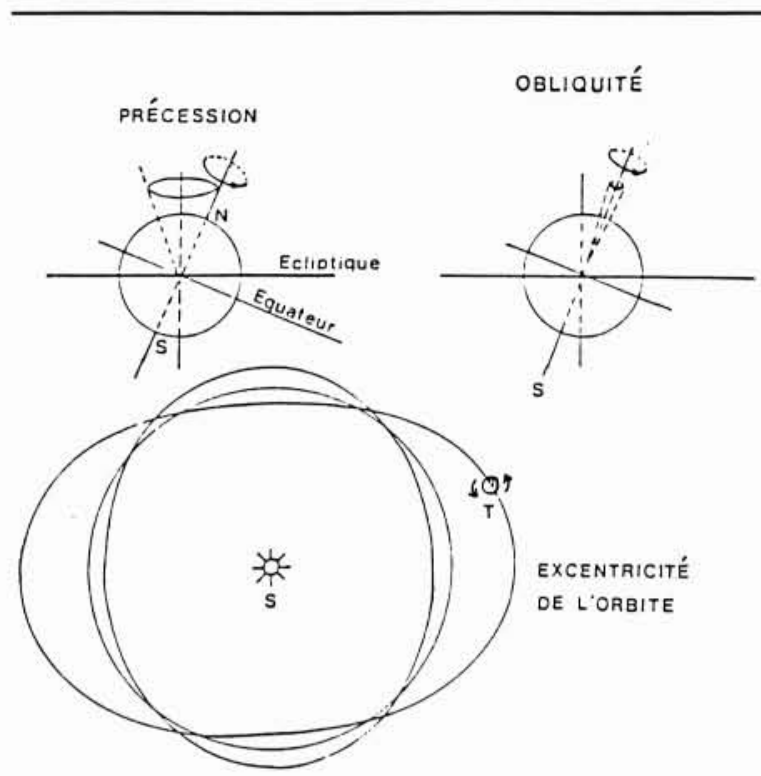

5. Les trois paramètres orbitaux de la terre. La combinaison de leurs variations périodiques est à la base du calcul des variations d'ensoleillement effectué par MiLANKOVITCH.
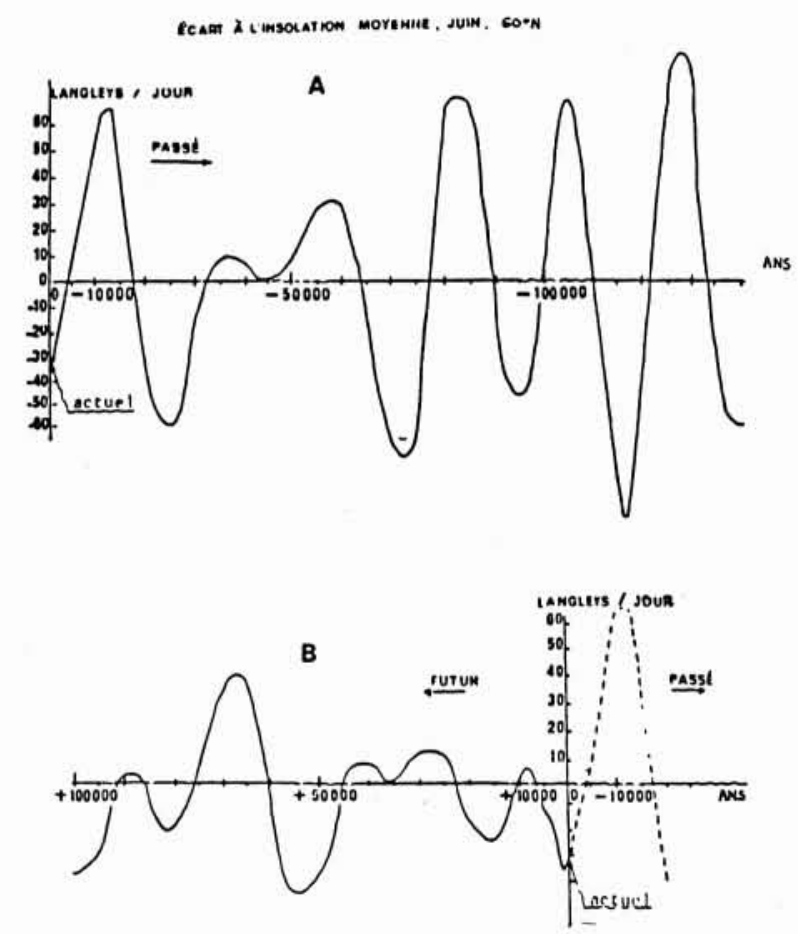

6. Variations d'insolation du passé et du futur d'après la théorie astronomique de MILANKOVITCH. Les variations, exprimées en langley/jour $\left(1\right.$ langley $=1$ calorie par $\left.\mathrm{cm}^{2}\right)$, sont des écarts à la valeur moyenne de l'insolation prise sur I million d'années. La courbe $\mathrm{A}$ représente pour le dernier cycle climatique les fluctuations d'insolation le 15 juin à la latitude $60^{\circ} \mathrm{N}$. En comparant cette courbe à celles des figures 7-8-9 qui donnent les dates des maxima de glaciation de ce dernier cycle, on voit que la correspondance est excellente. La courbe B représente les variations d'insolation dans le futur à la mi-juin et pour $60^{\circ} \mathrm{N}$ (d'après J. LABEYRIE, 1985). 

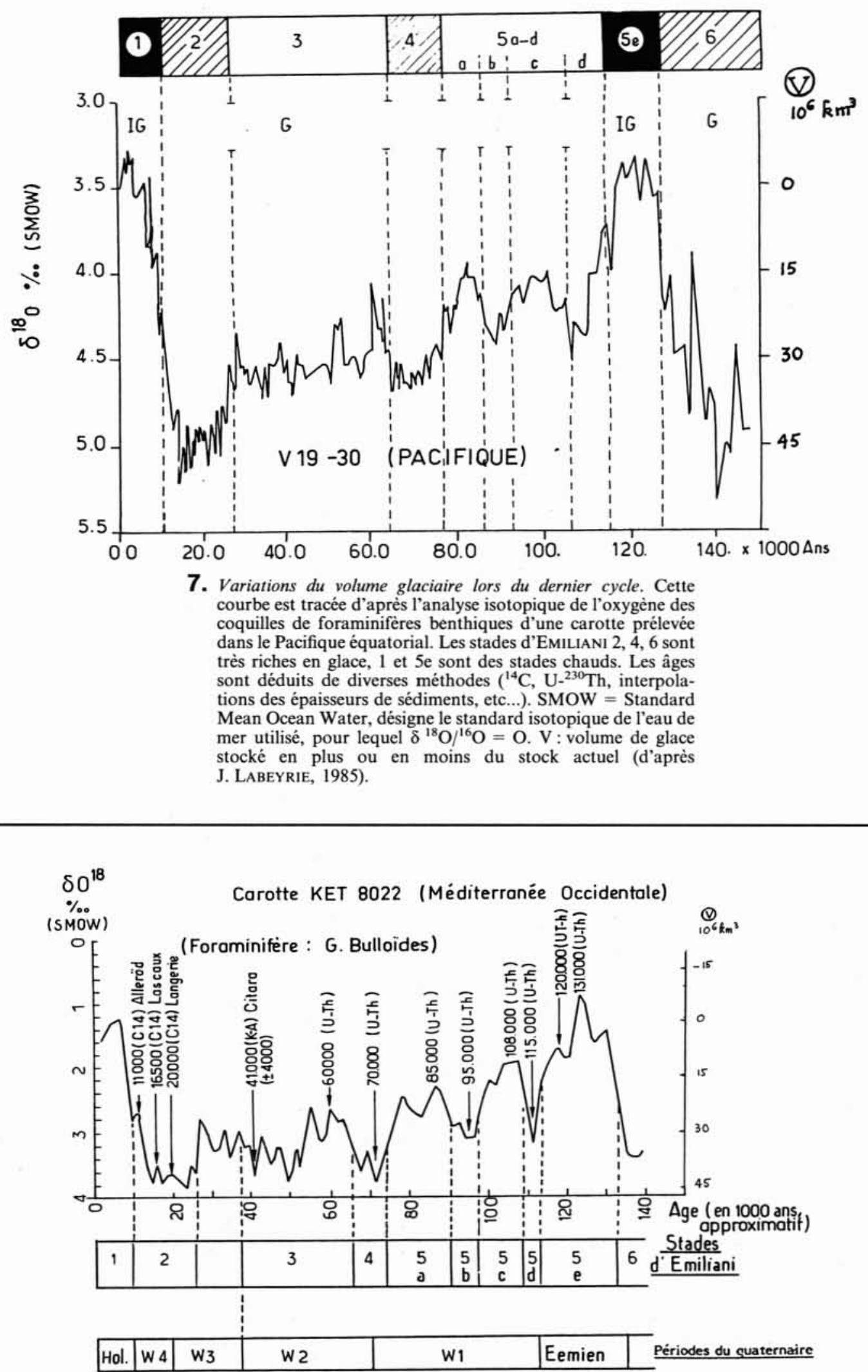

8. Variations climatiques du dernier cycle. A partir d'une carotte prélevée dans la Mer Tyrahénienne. Les âges et techniques de datation sont indiquées pour les principaux événements (maxima et minima) représentés (d'après J. LABEYRIE, 1985). 


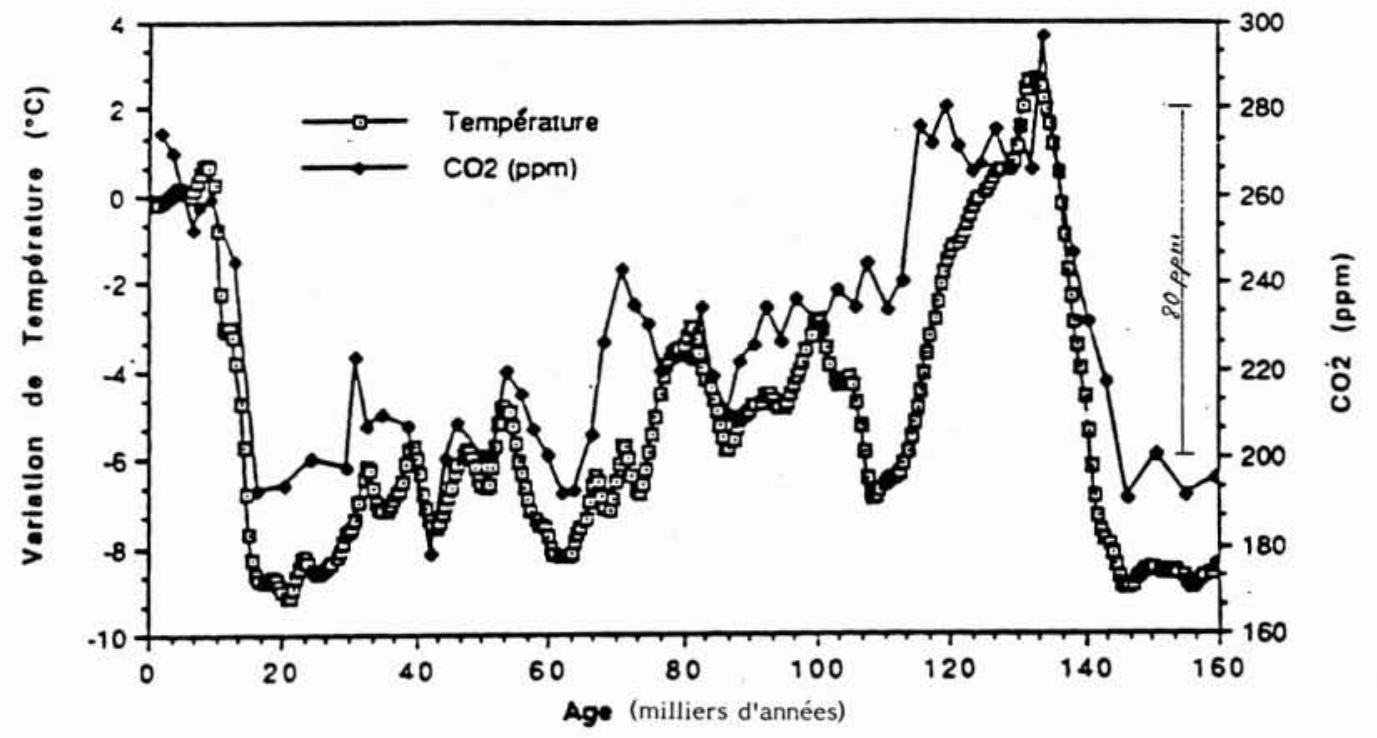

9. Variations de la température de l'air et de la teneur en gaz carbonique de l'atmosphère pendant le dernier cycle climatique. Les températures ont été déduites des variations de la composition isotopique de l'oxygène de la glace d'une carotte prélevée sur le site de Vostok (Antarctique). Les teneurs en $\mathrm{CO}_{2}$ ont été mesurées dans les bulles d'air piégées dans la glace (d'après C. LoRIUS et al., 1987).

10. Variation du niveau de la mer depuis 30000 ans. Cette variation a été reconstituée par la datation au ${ }^{14} \mathrm{C}$ de restes d'animaux et végétaux marins retrouvés aux divers niveaux fossiles de la mer. La courbe A a été tracée à partir d'échantillons prélevés devant les côtes d'Afrique tropicale et du Brésil, la courbe $\mathrm{B}$ à partir de coquilles prélevées au large des côtes du Languedoc. Ces 2 courbes coïncident parfaitement avec les courbes " isotopiques " donnant les variations du volume océanique (d'après J. LABEYRIE, 1985).

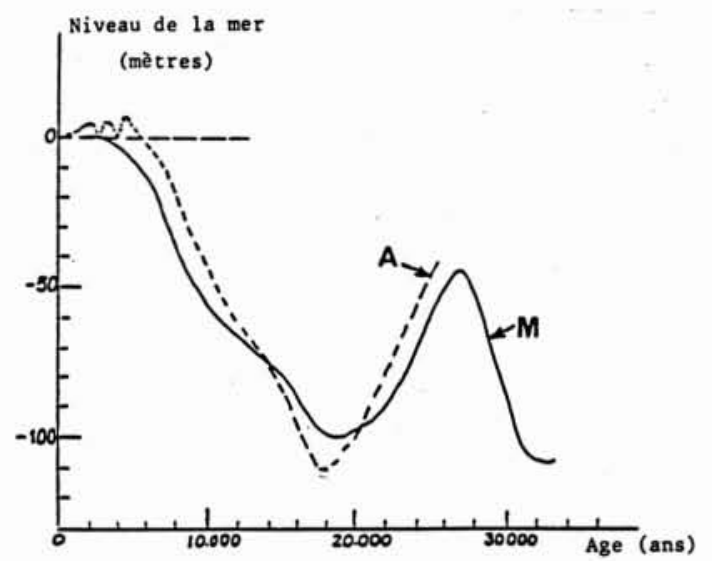

\section{Echelle de $\mathbf{1 0 0} 000$ ans : le dernier cycle climatique complet}

- Ce cycle est constitué d'une période " chaude » couvrant $65000 \mathrm{ans}$, approximativement de -140000 ans à 75000 ans, et d'une période " froide " pendant 50000 ans, de -75000 à -15000 ans correspondant au Würm des préhistoriens, qui a vu la formation des calottes glaciaires de l'hémisphère nord avec abaissement corrélatif du niveau marin. On constate, à l'intérieur de ces périodes, de nombreux accidents climatiques qui se traduisent par 5 oscillations majeures du niveau marin. Les figures 7-8-9 présentent les résultats d'analyse de 2 carottes de sédiments marins prélevés dans le Pacifique et en Méditerranée et d'une carotte de glace de l'Antarctique : ces 3 sources d'in- formations paléoclimatiques couvrent 140 à 160000 ans et révèlent les variations du volume glaciaire au cours de cette période pour les 2 premières carottes et les variations de la température et de la concentration en $\mathrm{CO}_{2}$ de l'air pour la $3^{\text {c }}$. Une coïncidence remarquable apparaît entre les structures climatiques mises en évidence à l'échelle planétaire par ces différents indicateurs.

- Qui dit stockage continental de glaces dit abaissement du niveau marin : les variations reconstituées sur 30000 ans à partir des analyses de débris végétaux et animaux de rivages fossiles, prélevés au large des côtes d'Afrique Occidentale, du Brésil et du Languedoc, révèlent encore ici un excellent accord (fig. 10). On retiendra que le niveau actuel de la mer s'est stabilisé, à quelques oscillations mineures près, il y a seulement environ 7000 ans. 


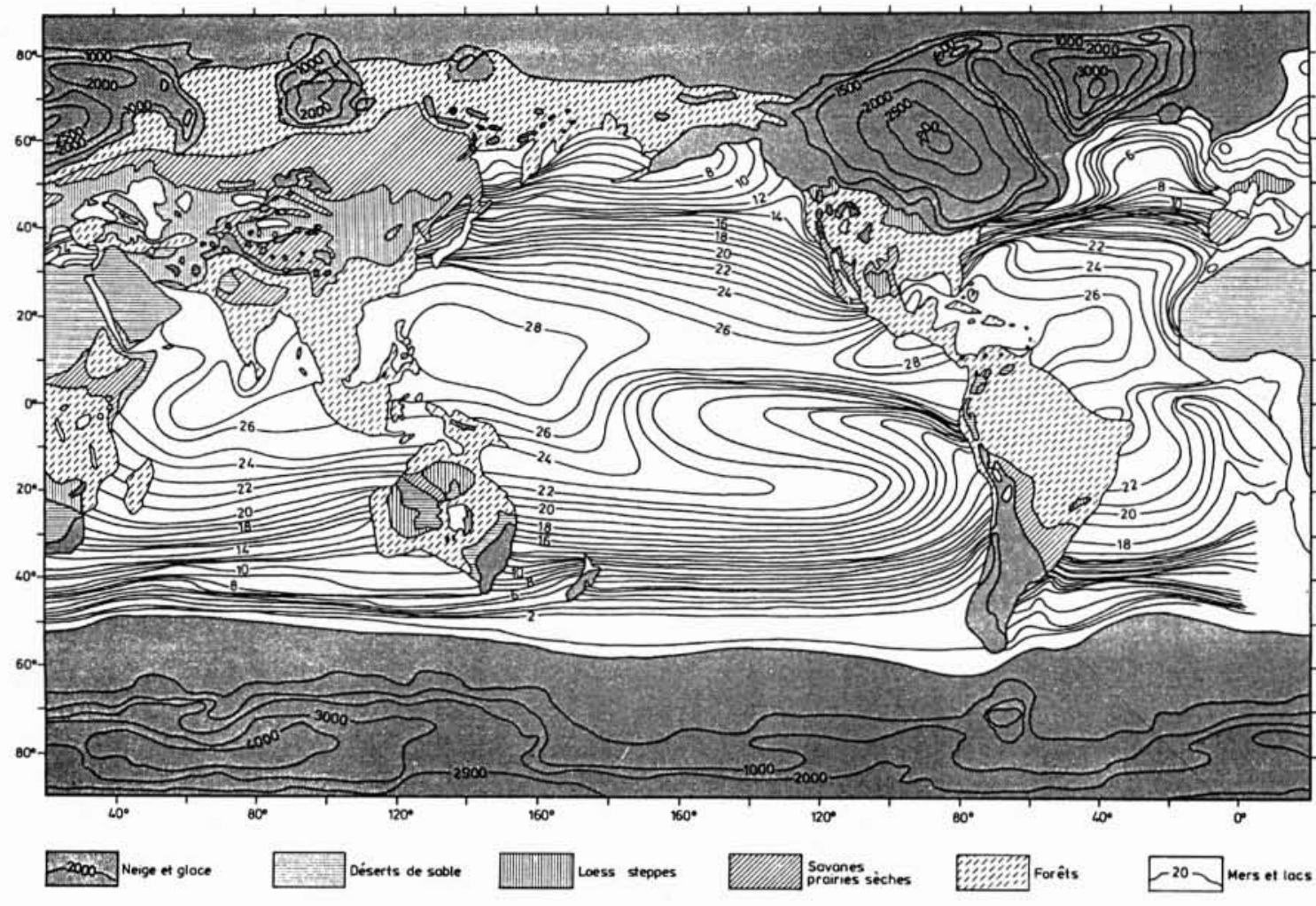

11. Carte de répartition des températures marines, des glaces, de la végétation des étés vers $18000 \mathrm{BP}$. La carte représente aussi le contour des continents correspondant au niveau marin $-120 \mathrm{~m}$ en dessous du niveau actuel. Les épaisseurs des calottes glaciaires continentales sont indiquées (d'après Climar, 1976)

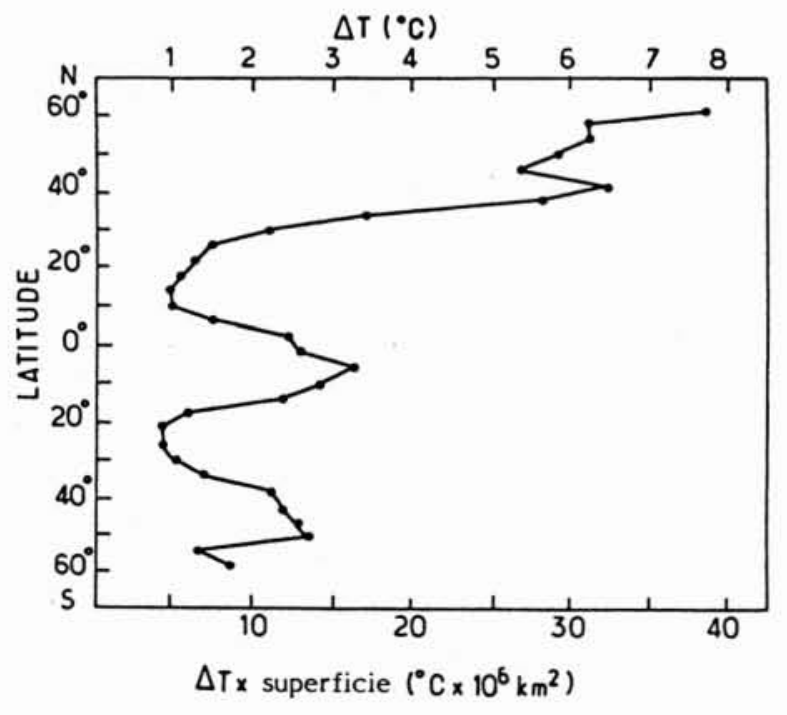

12. Différences moyennes des températures moyennes d'été des surfaces marines, par zones de latitudes entre 18000 BP et aujourd'hui (d'après CLIMAP, 1976).

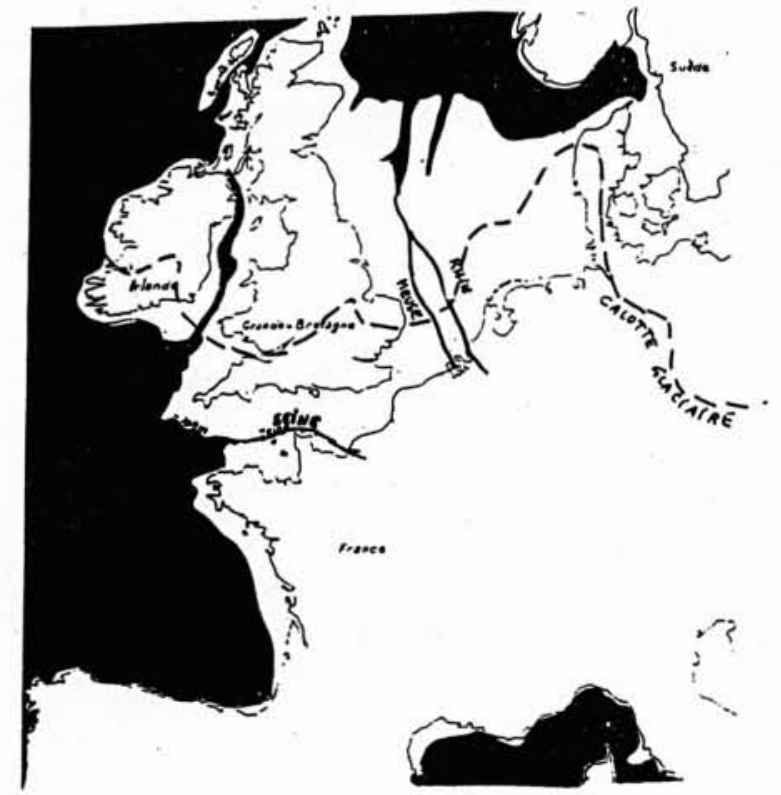

13. Les rivages de l'Europe de l'Ouest vers $18000 \mathrm{BP}$ au moment du maximum de développement des calottes glaciaires sur le Nord de l'Europe et sur le Canada (d'après J. LABEYRIE, 1985). 


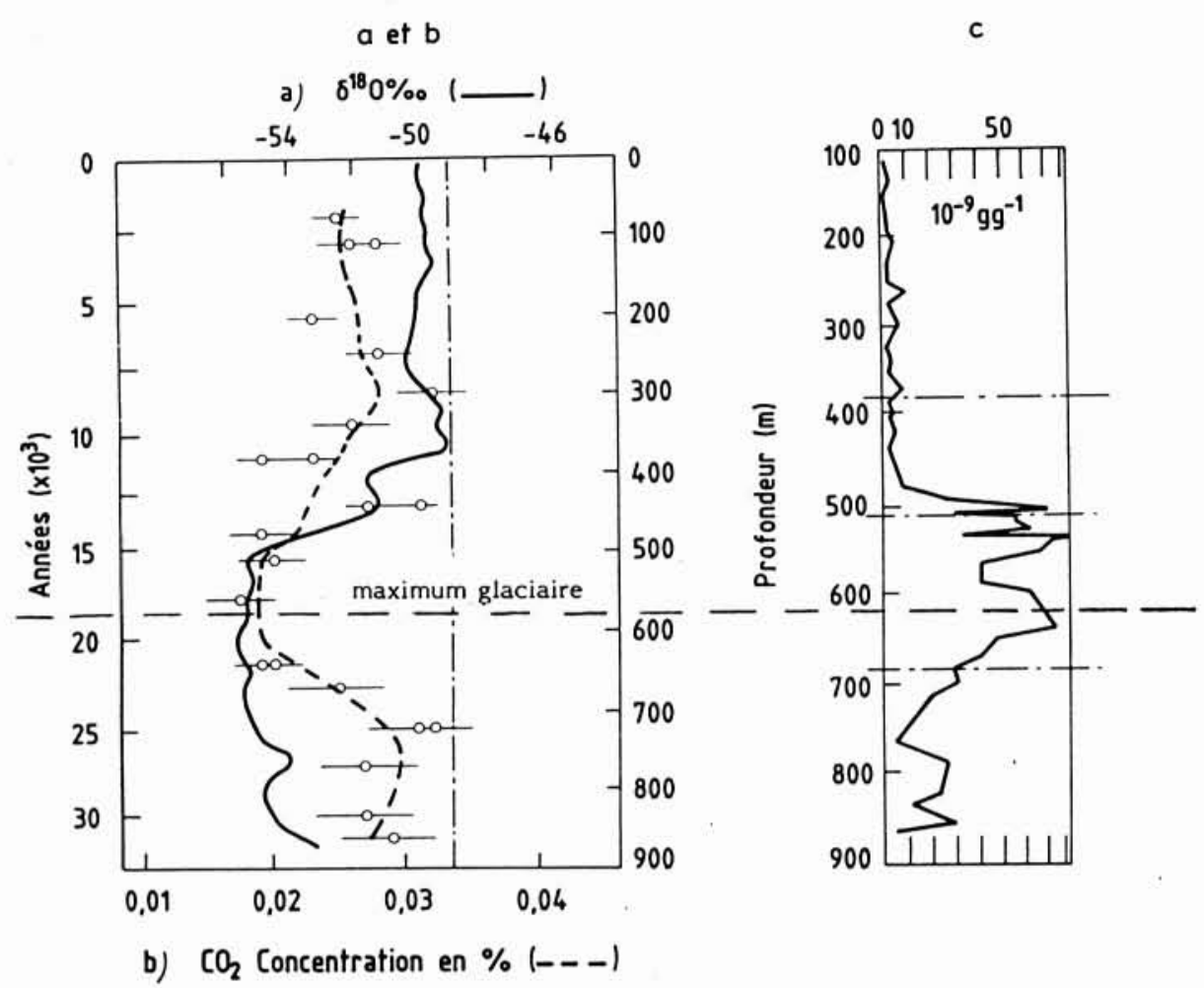

14. Résultats de l'analyse de la carotte de glace du Dôme C $\left(75^{\circ}\right.$ Sud, Antarctique) (d'après C. Lorius, 1983).

courbe a : composition isotopique de l'oxygène de la glace, courbe b: concentration en $\mathrm{CO}_{2}$ de l'atmosphère, courbe $\mathrm{c}$ : abondance des poussières continentales dans la glace (variations de l'aluminium, élément caractéristique des poussières continentales).

- Le dernier paroxysme glaciaire a eu lieu vers - 18 ou - 20000 ans et cette période est maintenant bien documentée. On sait que le désert saharien recouvrait alors le Sahel, toutes les périodes glaciaires se traduisant par une désertification très poussée et un fort déficit pluviométrique. A ce moment-là le volume des glaces continentales s'élève à 47 millions de $\mathrm{km}^{3}$, soit 2 fois le volume actuel.

- Au cours de la décennie 1970 , le programe international CLIMAP a permis de reconstituer la température de surface de toutes les mers du globe au mois d'août $18000 \mathrm{BP}$ avec une précision de l'ordre de $1,5^{\circ} \mathrm{C}$ et cela grâce à l'analyse de groupements de foraminifères. On a pu tracer ainsi la carte météorologique de "l'été $18000 \mathrm{BP}$ » (fig. 11) sur laquelle figurent les isothermes des températures marines, le contour des continents alors que le niveau marin était à environ - $120 \mathrm{~m}$ par rapport au niveau actuel, et la répartition des couvertures continentales: glaces, déserts, végétation déduite des analyses palynologiques. Les différences de ces températures avec les températures marines de surface actuelles par zones de latitudes (fig. 12) sont faibles sous les tropiques et beaucoup plus fortes aux hautes latitudes, qui constituent des régions particulièrement sensibles: on retrouvera cette configuration lorsque l'on tente d'évaluer les conséquences thermiques planétaires d'un doublement de la concentration atmosphérique du
$\mathrm{CO}_{2}$. La carte des rivages de l'Europe de l'Ouest de 18000 ans (fig. 13) montre que la Manche et la Mer du Nord sont à sec, cette dernière en grande partie recouverte par la calotte glaciaire. Le plateau continental est parcouru par le prolongement des fleuves actuels Rhin-Meuse et Seine et la calotte glaciaire recouvre en quasi totalité l'Irlande, la Grande-Bretagne et l'Allemagne Orientale.

- En ce qui concerne les variations de composition de l'atmosphère en gaz à effet de serre, on constate une assez bonne coïncidence entre les variations de concentrations de $\mathrm{CO}_{2}$ et les périodes froides et chaudes; lors du maximum de la dernière glaciation, la concentration atmosphérique du $\mathrm{CO}_{2}$ a diminué d'environ $1 / 3$ et s'est abaissée à 200 ppmv! (fig. 14). On constate également qu'au moment de ce maximum glaciaire les poussières d'origine continentale ont augmenté d'un facteur 20 dans la glace antarctique : on peut avancer ici que les transports atmosphériques de poussières sont liés à la raréfaction de la végétation et que cet afflux correspond à une certaine désertification. Cette dénudation, jointe à l'accroissement des surfaces enneigées aux hautes latitudes, a dû produire dans les régions tempérées une augmentation de l'albedo qui vint ajouter son effet réfrigérant à celui résultant de la présence de grandes calottes polaires plus au nord. 
- La très grande sensibilité du cycle hydrologique aux variations climatiques apparaît à travers deux informations :

- on a récemment montré, à partir des associations de végétaux déduites de l'analyse des pollens d'une carotte de sédiments continentaux prélevés dans la région lyonnaise, qu'à l'époque du dernier maximum glaciaire les précipitations (pluies et neige) reçues par cette zone ne représentaient que 200 à $250 \mathrm{~mm}$ par an, soit le quart de la pluviosité actuelle ;

- l'analyse des dépôts de sédiments d'origine continentale prélevés dans le Nord de l'Océan indien et les mesures isotopiques sur les coquilles de foraminifères qu'ils contiennent, ont permis de montrer au maximum glaciaire une diminution d'un facteur 2 du débit des grands fleuves qui drainent le Sud-Est Asiatique : la mousson d'été y a pratiquement disparu ! Il s'agit là d'un indice supplémentaire des modifications très profondes qui ont affecté les circulations atmosphérique et océanique.

Ces quelques coups de projecteur suffisent à mettre en valeur la variété, la quantité et la qualité des informations dont on dispose aujourd'hui sur ce dernier cycle climatique et son maximum glaciaire.

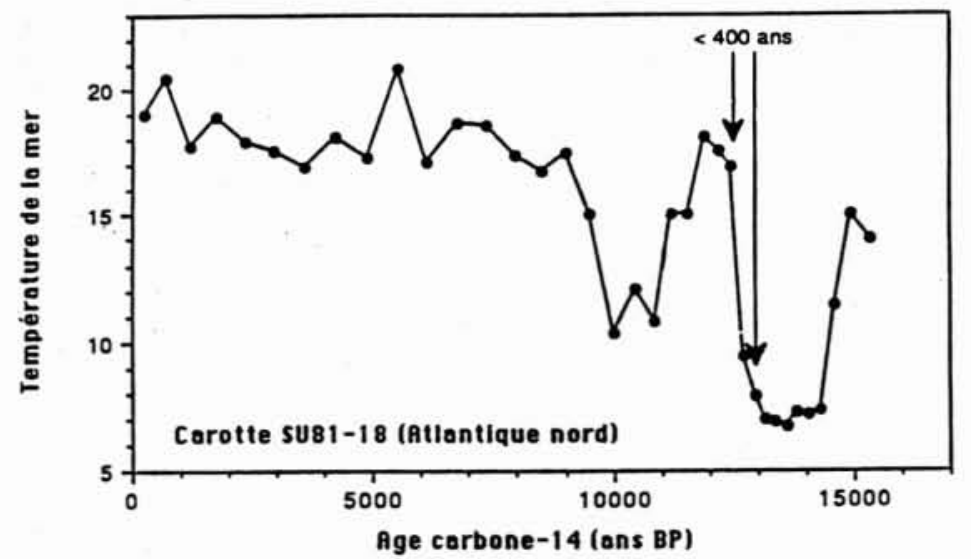

15. Variations de la température moyenne anmuelle de l'eau de surface dans l'Océan Atlantique au large du Portugal déduites des variations de faune dans les sédiments avec datation au ${ }^{14} \mathrm{C}$ des foraminifères. On notera la brutalité du réchauffement de l'ordre de $10^{\circ} \mathrm{C}$ en un temps inférieur à 400 ans vers 12500 BP (d'après J.C. DUPLESSY et al., 1987).

Tableau 2. Variations de la température de surface de la mer en été au large de l'Europe de l'ouest et remontée du niveau marin au début du cycle climatique actuel.

\begin{tabular}{|c|c|c|c|c|c|c|}
\hline \multirow{2}{*}{$\begin{array}{l}\text { Dates } \mathrm{C}-14 \\
\text { non rectifiés } \\
\text { (ans BP) }\end{array}$} & \multirow{2}{*}{$\begin{array}{l}\text { Niveau } \\
\text { de la mer } \\
\text { (mètres) }\end{array}$} & \multicolumn{4}{|c|}{$\begin{array}{l}\text { Températures d'été de surface de la mer } \\
\left(\text { en }^{\circ} \mathrm{C}\right) \text { au large de }\end{array}$} & \multirow{2}{*}{$\begin{array}{c}\text { Périodes du } \\
\text { quaternaire récent } \\
\text { et } \\
\text { (repères archéologiques) }\end{array}$} \\
\hline & & Irlande & Quimper & Nantes & Bordeaux & \\
\hline 0 & 0 & 14 & 16,5 & 17 & 20 & \multirow{8}{*}{ Maximum climatique chaud } \\
\hline 2000 & +2 & 15 & 16,2 & 16 & 18 & \\
\hline 2800 & 0 & 15 & 15 & 15,5 & 15 & \\
\hline 3500 & +3 & 15 & 16 & 18 & 19 & \\
\hline 4000 & 0 & 16 & 16 & 20 & 20 & \\
\hline 5400 & $+4,5$ & 16,5 & 16,5 & 20 & 22 & \\
\hline 7000 & 0 & 16,5 & 16 & 18 & 21 & \\
\hline 8000 & -12 & 14,5 & 15 & 20 & 22 & \\
\hline 9500 & -40 & 12 & 10 & 10 & 13 & \\
\hline 10000 & -45 & 10 & 7 & 7 & 10 & (PInCEVEnt) \\
\hline 11300 & -60 & 13,5 & 20 & 21 & 20 & \multirow[b]{2}{*}{ WÖRM IV } \\
\hline 13000 & & 12 & 18 & 20 & 18 & \\
\hline 15000 & -110 & 7 & 6 & 6 & 6 & \\
\hline 16500 & -100 & 8 & 7 & 7 & 7 & \multirow[t]{2}{*}{ (LASCAUX) } \\
\hline 18000 & -120 & & & & & \\
\hline
\end{tabular}

Les variations de températures sont déduites des variations de mélanges de populations de foraminifères dans 4 carottes prélevées dans l'Atlantique au large de l'Islande, de la Bretagne et dans le Golfe de Gascogne. Les variations de niveau de la mer sont déduites de mesures de côtes fossiles en Méditerranée et au Brésil (pour la période 7000 BP-actuel) (d'après J. LABEYRIE, 1984). 


\section{Echelle de 10000 ans : le réchauffement spectacu- laire accompagnant l'essor de la civilisation néolithique}

- Le réchauffement qui marque la fin du dernier cycle et le début du cycle actuel s'est traduit par une remontée spectaculaire du niveau marin, passant de $-120 \mathrm{~m}$ au maximum glaciaire de $18000 \mathrm{BP}$, jusqu'au niveau actuel atteint vers $7000 \mathrm{BP}$. Quelques données sont indispensables à la description de ce phénomène qui ne s'est pas déroulé sans soubresauts, oscillations et accélérations. La paléoclimatologie associée à la palynologie et à l'archéologie préhistorique fournissent ici des quantités d'informations qui attendent des explications.

- Le Würm IV des préhistoriens couvre cette période contrastée, marquée par des épisodes très chauds et très froids, jalonnée de repères archéologiques : l'épisode chaud de LASCAUX vers $16500 \mathrm{BP}$, le début de la civilisation néolithique et son essor avec les indices formels d'agriculture, d’élevage et de sédentarité qui vont se multiplier à partir de $8000 \mathrm{BP}$, les chasseurs de rennes à PINCEVENT au bord de la Seine lors d'un refroidissement brutal vers $10000 \mathrm{BP}$, atteignant au début de l'Holocène des valeurs de températures analogues à celles du maximum glaciaire de $18000 \mathrm{BP}$ mais dont la durée ne dépassera pas le millénaire, etc...

- On note l'apparition de véritables phénomènes " transitoires " , tel le réchauffement brutal de la température de surface de l'Atlantique nord de $10{ }^{\circ} \mathrm{C}$ en moins de 400 ans constaté entre 13000 et $12000 \mathrm{BP}$ (fig. 15), le " coup de froid" de 11000 à 10000 BP déjà cité, les "coups de chaleur " qui amèneront des températures de surface de la mer en été supérieures à la température actuelle de plus de $2{ }^{\circ} \mathrm{C}$ vers $12000 \mathrm{BP}$ et entre 6000 et $5000 \mathrm{BP}$ au moment où va s”établir le "climax" ou optimum climatique de ce réchauffement, avec l'épanouissement en Europe de la " Chênaie mixte " (chêne-orme-tilleul-noisetier) caractéristique du climat "Atlantique " sous la dépendance de la chaleur distribuée par le Gulf Stream. Après ce maximum de la période chaude, à travers la succession de hauts et de bas, on perçoit de façon générale un lent refroidissement de l'Holocène récent (cf. Tableau 2).

- La vitesse de remontée du niveau marin a été très variable, dépassant sans doute $2 \mathrm{~m}$ par siècle sur des durées de l'ordre ou inférieures au millénaire. Rapportée à l'ensemble du phénomène, donc sur 11000 ans, elle est de $1 \mathrm{~m}$ par siècle en moyenne, ce qui correspond à la fusion de 3600 $\mathrm{km}^{3}$ de glaces continentales par an. Ce processus absorbe une énergie équivalent à $0,8 \mathrm{~W} \cdot \mathrm{m}^{-2}$, soit $0,03 \%$ de l'énergie du rayonnement solaire absorbée en moyenne annuelle par le système Terre-Atmosphère. Ces chiffres suffisent à montrer combien une variation de tendance, somme toute minime sur le plan quantitatif mais poursuivant son action pendant une longue période, produit en fin de compte un énorme changement non seulement géographique mais aussi climatique à échelle planétaire. Ceci incite encore plus fortement à rechercher les causes responsables et les mécanismes explicatifs de ce réchauffement.
- La déglaciation des continents peut être suivie grâce à la datation au carbone-14 des moraines qui jalonnent le retrait des calottes (fig. 16 et 17, page suivante). En comparant les surfaces couvertes de glace à chaque époque aux indications des volumes stockés déduits des courbes isotopiques, on obtient les épaisseurs de glaces correspondantes, qui ont dépassé $3000 \mathrm{~m}$ au centre des inlandsis lors des maxima glaciaires.

- Au cours de cette déglaciation continentale, le Sahara a connu une période humide qui s'est traduite par un recul du désert entre 12000 et $6000 \mathrm{BP}$. En particulier il s'est couvert de nombreux lacs, qui ont pu être datés à partir de leurs dépôts fossiles, entre 12000 et $9000 \mathrm{BP}$. Un indice de l'intensité de cette grande période pluviale est fourni par l'analyse de carottes de sédiments prélevés au débouché du delta du Niger : on met en évidence entre les années 12000 et $10000 \mathrm{BP}$ une énorme augmentation du flux de sédiments charriés par le fleuve, correspondant à une érosion hydrique intense au Sahel.

- On notera enfin qu'au cours de cette remontée du niveau marin, la concentration du $\mathrm{CO}_{2}$ atmosphérique s'accrôt d'environ 80 ppmv, soit d'une quantité équivalente à l'accroissement observé depuis l'ère industrielle. Il faut ajouter que, dans un cas, on part de 200 ppmv et, dans l'autre, de 270 ppmv comme concentrations de départ et que les rythmes de croissance diffèrent foncièrement d'un facteur 100 ! Le fait vaut d'être noté dans l'attente d'explications sur les causes et effets de cet accroissement.

\section{Echelle de 1000 ans : la relative "stabilité " du climat planétaire n'exclut pas des variations signi- ficatives au cours de la période historique}

Trois exemples ont été choisis pour illustrer ces variations :

- Les niveaux marins après avoir atteint le niveau actuel vers $7000 \mathrm{BP}$ ont continué à croître pour culminer à $+4.5 \mathrm{~m}$ vers $5300 \mathrm{BP}$ : il y eut ensuite 2 oscillations amorties avec des maxima secondaires à $+3 \mathrm{~m}$ vers $3400 \mathrm{BP}$ et à $+2 \mathrm{~m}$ vers $2300 \mathrm{BP}$ avant un retour progressif au niveau actuel. Ces oscillations du niveau marin sont à mettre en relation avec l'immersion des atolls du Pacifique au-dessus de la cote $+2.5 \mathrm{~m}$ et surtout avec l'émergence $\mathrm{du}$ delta du Nil au-dessous du niveau $+3 \mathrm{~m}$ qui commença vers $4700 \mathrm{BP}$ (fig. 18, page suivante). Lorsque la majeure partie de sa surface actuelle se trouva exondée vers $4200 \mathrm{BP}$, la conquête du Delta vers les rois du Sud amorça le développement de la civilisation dynastique égyptienne.

- la pluviométrie saharienne, après les périodes humides que l'on a évoquées, a connu depuis 7000 BP une régression générale allant de pair avec le processus de désertification. Des lacs sahariens, seul subsiste aujourd'hui le lac Tchad dont la chronologie des fluctuations, rétractions et extensions temporaires, est un indicateur précieux des variations pluviométriques au Sud du Sahara et dans le Sahel (fig. 19, page suivante). On constate ainsi, notamment entre 3000 et $2000 \mathrm{BP}$, une extension du lac à 


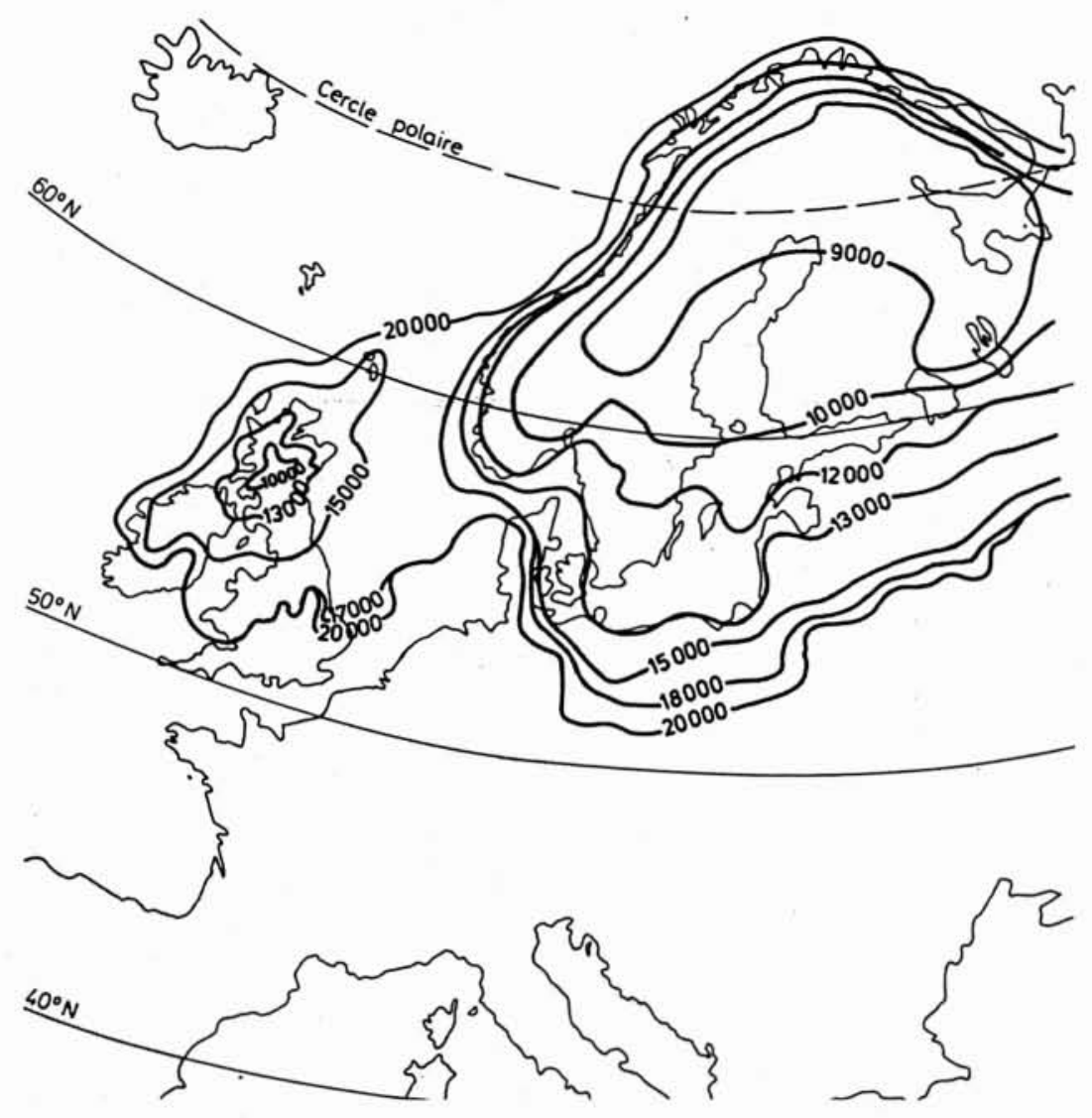

16. La déglaciation sur l'Europe du Nord, de 20000 BP à 9000 BP (d'après J. LABEYRIE, 1984).

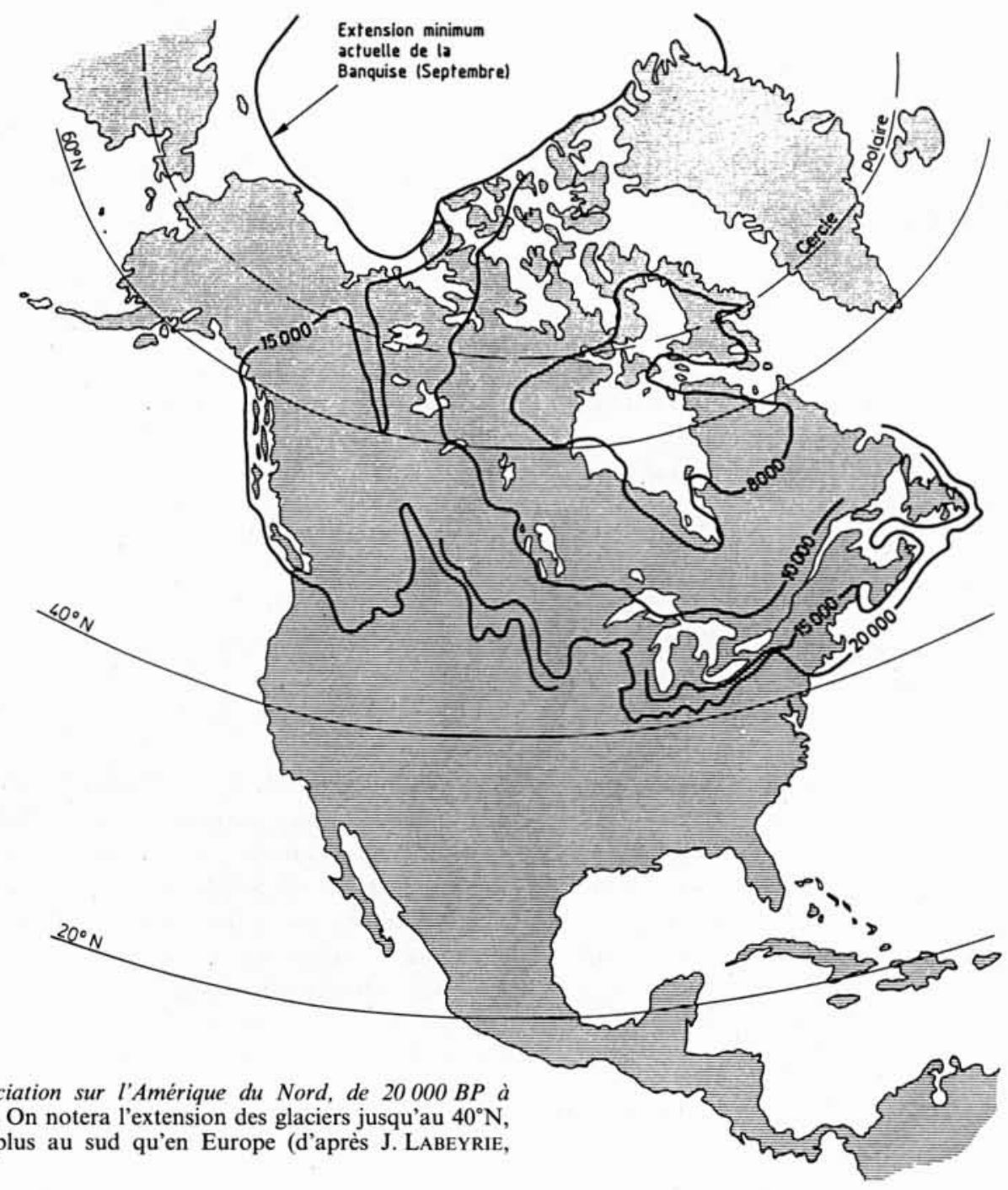
$8000 B P$. On notera l'extension des glaciers jusqu'au $40^{\circ} \mathrm{N}$, soit $12^{\circ}$ plus au sud qu'en Europe (d'après J. LABEYRIE, 1984). 


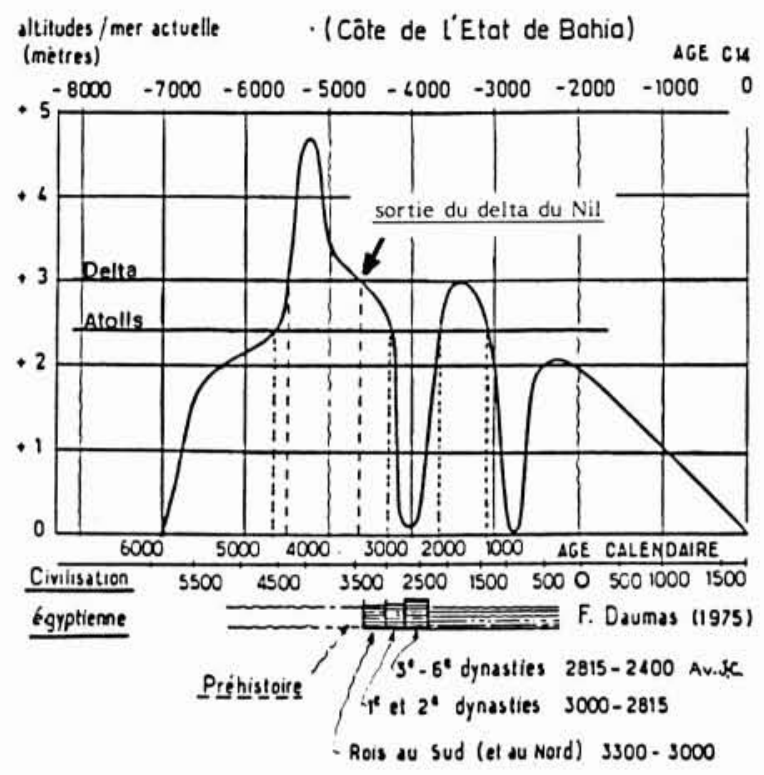

18. Oscillations du niveau marin depuis 7000 ans. Les 3 oscillations culminant à $+4,5 \mathrm{~m},+3 \mathrm{~m}$ et $+2 \mathrm{~m}$ ont été découvertes sur les côtes du Brésil et leur âge déterminé par datation au ${ }^{14} \mathrm{C}$ des dépôts qu'elles avaient laissé (d'après J. LABEYRIE, 1985).

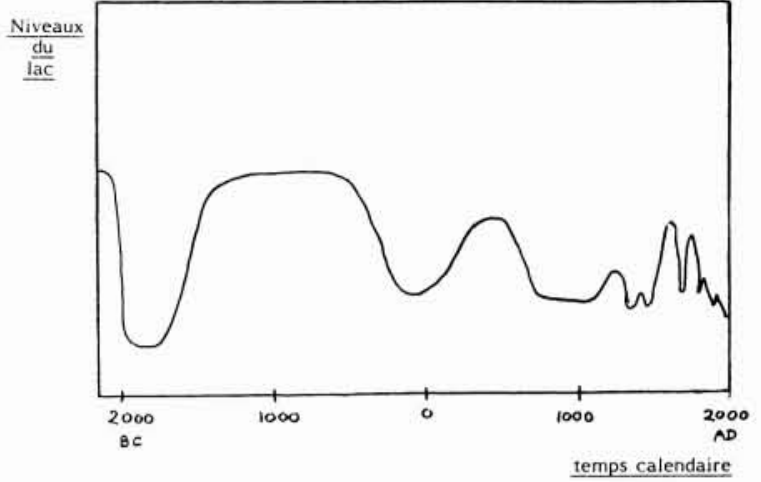

19. Fluctuations des niveaux du lac Tchad au cours des 4 derniers millénaires (d'après S. NichOLSON, 1980).

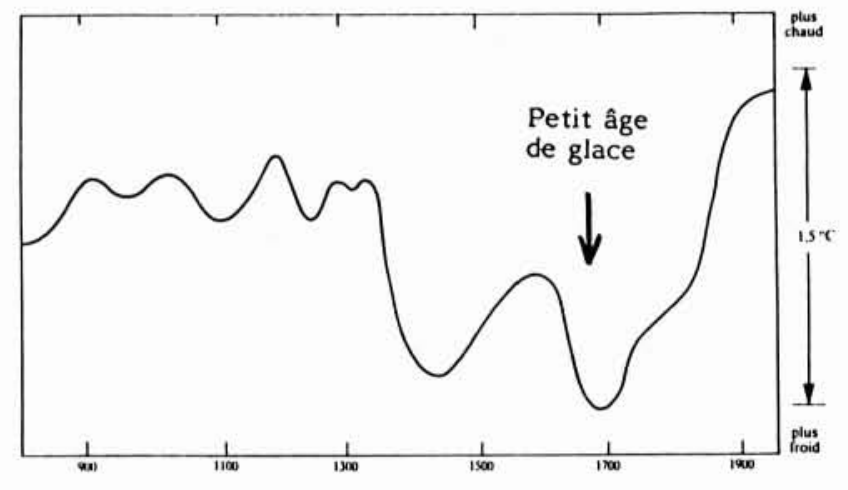

20. Fluctuations climatiques récentes au cours du dernier millénaire. On remarquera le minimum du $\mathrm{XVII}^{\mathrm{e}}$ et $\mathrm{XVIII^{e }}$ siècles correspondant au "petit âge glaciaire " (d'après I. RASOOL et N. SKROTZKY, 1988). plusieurs centaines de $\mathrm{km}$ du périmètre actuel qui correspond à un regain de climat humide en relation avec une légère remontée du niveau marin. On retiendra, des constats effectués aux diverses échelles de temps, que le Sahara est humide quand les calottes glaciaires fondent et redevient désert durant les périodes froides : on l'a vérifié au moins pour les 7 derniers cycles.

- Les fluctuations de températures au cours du dernier millénaire, déduites de différents indices ou témoignages, et dont l'amplitude n'excède pas $+1.5^{\circ} \mathrm{C}$, ont suffi à provoquer des désastres autant dûs au froid qu'aux étés pluvieux destructeurs de récoltes. Les grandes lignes de ces fluctuations font apparaître du $\mathrm{VIII}^{\mathrm{e}}$ au XII ${ }^{\mathrm{e}}$ siècle un optimum climatique avec toutefois autour de l'an 1000 une pluviosité entraînant famine et désastres. Du XII ${ }^{\mathrm{e}}$ au XIV ${ }^{\mathrm{e}}$ siècle, une nouvelle avancée des glaciers est suivie d'un adoucissement avec hivers humides, et la $2^{\mathrm{c}}$ moitié du $\mathrm{Xv}^{\mathrm{e}}$ siècle voit une amélioration climatique : c'est l'époque des grandes navigations exploratoires autour du monde. Entre 1550 et 1850 , se situe une avancée glaciaire correspondant $\mathrm{au}$ "petit âge de glace " qui culmine en Europe aux XVII et $\mathrm{XVIII}^{\mathrm{e}} \mathrm{s}$. bien qu'entrecoupée de périodes tempérées (fig. 20). A son paroxysme, ce "petit âge glaciaire" se situe à $-3{ }^{\circ} \mathrm{C}$ seulement en dessous du maximum (optimum) thermique de la planète évoqué plus haut (vers $6000 \mathrm{BP})(*)$.

(*) J'ai relevé un témoignage sur ce "petit âge de glace » dans les registres paroissiaux de Chirac-l'Eglise (aujourd'hui Chiratl'Eglise) dans l'Allier où le curé GALDET écrit : "en l'année 1766 , le 21 décembre la gelée commença et ne discontinua d'un moment jusqu'à Pâques qui était le 19 d'avril 1767 (...). Cette gelée fut si forte que la plupart des arbres en furent fendus. Le vin se gelait en peu de minutes dans le calice sur l'autel. Il fallait avoir des réchaux pour poser près du calice sur l'autel pour éviter la congélation du vin (...). La gelée de l'hiver 1768 fut aussi violente et même plus que la précédente année, mais elle ne dura que pendant une vingtaine de jours (...). Dans le courant de l'année 1769, la misère était si grande et les pauvres si nombreux qu'on n'osait plus sortir dans la crainte de trouver des gens affamés dans les chemins... " 
$\mathrm{CO2}$ (ppm)

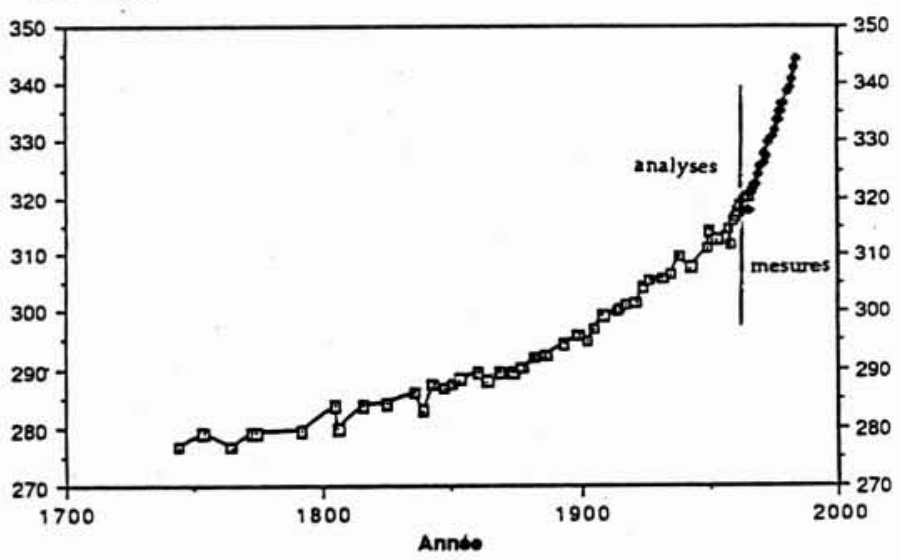

21. Variations de la teneur en gaz carbonique de l'atmosphère depuis 1744.

- Variations de 1744 à 1965 déduites de l'analyse de l'air piégé dans la glace antarctique.

- Variations à partir de 1965 mesurées en continu au site de Mauna LoA (Hawaī).

On remarquera que la perturbation du cycle atmosphérique du carbone après l'ère industrielle est du même ordre de grandeur $(70 \mathrm{ppmv})$ que celle associée au passage d'une période glaciaire à une période interglaciaire (cf. fig. 9).

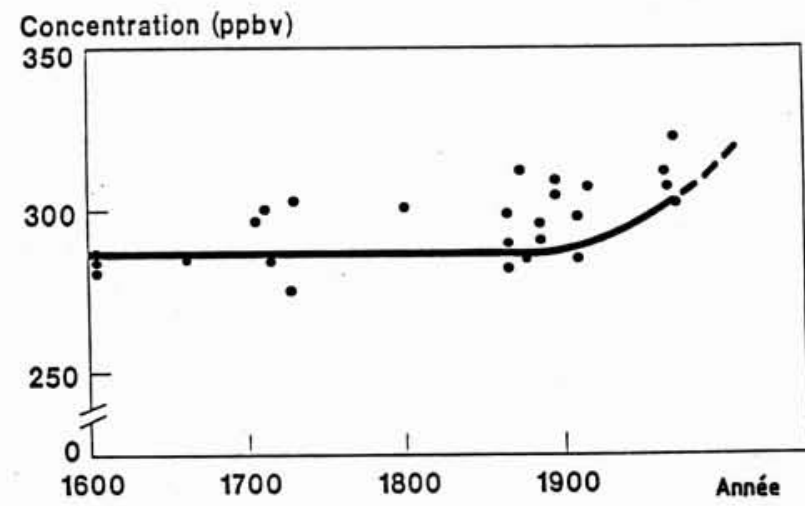

22. Variations de la teneur en oxyde nitreux $\mathrm{N}_{2} \mathrm{O}$ de l'atmosphère depuis 1600.

- Variations déduites de l'analyse de l'air piégé dans la glace.

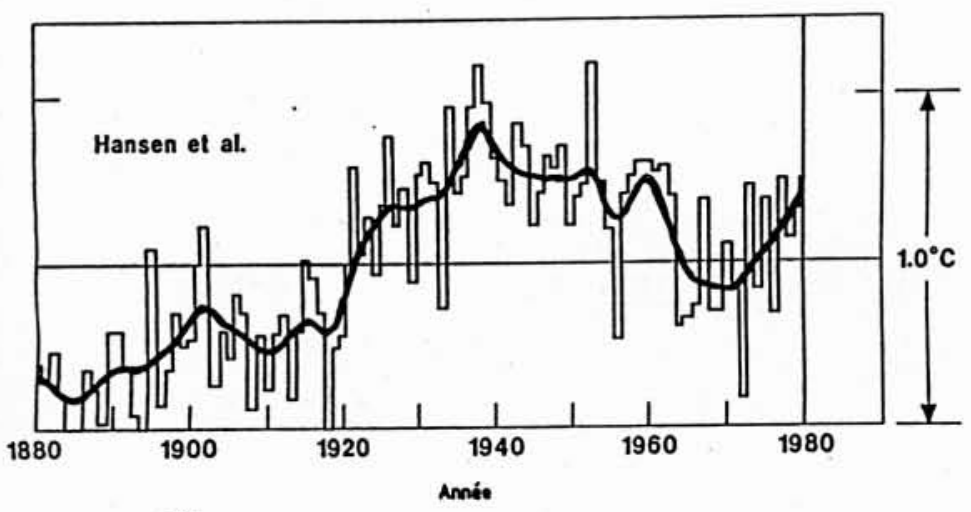

23. Variations de la température de l'air de l'Hémisphère Nord de 1880 à 1980.
5. Echelle du siècle: se pose ici le prolème de l'influence potentielle de l'homme sur le climat planétaire

- Trois voies sont possibles pour modifier le bilan radiatif et thermique du système Terre-Atmosphère qui conditionne le climat planétaire :

- les altérations de composition de l'atmosphère en espèces minoritaires qui affectent sa fonction "filtre " vis-à-vis des rayonnements solaire et terrestre et notamment son effet de serre qui joue un rôle essentiel dans le bilan thermique de la biosphère ;

- les modifications de l'albedo de la surface terrestre, qui conditionne l'absorption du rayonnement solaire;

- les rejets de chaleur sensible et latente, du fait des activités humaines, qui affectent directement les transferts d'énergie régissant la cirulation atmosphérique et le cycle de l'eau.

- Ces deux dernières voies conduisent à des modifications climatiques locales ou régionales (par exemple, le phénomène d'" îlot chaud " urbain) et ne sont pas susceptibles de provoquer des effets de dimension planétaire en raison des ordres de grandeur des modifications introduites par l'action de l'homme. Il n'en va pas de même avec la $1^{\text {re }}$ voie et l'on assiste depuis le début de l'ère industrielle à une modification significative de la composition de l'atmosphère par l'accroissement des concentrations de gaz à effet de serre: $\mathrm{CO}_{2}, \mathrm{CH}_{4}, \mathrm{~N}_{2} \mathrm{O}$, etc..., accroissement en relation directe avec le développement de certaines activités humaines (fig. 21-22).

- Le problème de l'identification de l'impact climatique de ces altérations est d'ores et déjà posé : il s'agit d'identifier un signal par rapport au "bruit de fond " des fluctuations " naturelles" de différents indicateurs climatiques choisis au niveau planétaire : températures, niveaux marins, paramètres du cycle hydrologique.

- Au cours du siècle 1880-1980, on constate une tendance globale au réchauffement qui se situe à l'intérieur d'un intervalle d'amplitude $+1{ }^{\circ} \mathrm{C}$ (fig. 23). Une analyse plus fine des températures de l'air observées sur les continents de l'hémisphère Nord fait apparaître des alternances de refroidissements (1880-1890, 1940-1970) et de réchauffements (1890-1940 et après 1970).

- L'évolution du niveau marin au cours du siècle passé (fig. 24) accuse une croissance moyenne de l'ordre du millimètre par an intégrant les phénomènes suivants: mouvements eustatiques de la croûte terrestre, fonte des glaciers continentaux, expansion thermique des océans, variations des réserves de glace de l'Antarctique et du Groenland, mais leurs contributions respectives à cette croissance sont mal définies tant en valeur absolue qu'en signe. Deux questions se posent à propos de cet indicateur fondamental de changement du climat planétaire :

- la définition des variations du niveau marin moyen sur une longue période, en raison des difficultés d'appréhension de faibles variations à partir du traitement complexe d'observations locales nécessitant le filtrage des effets de la 
Niveau moyen (en ca)

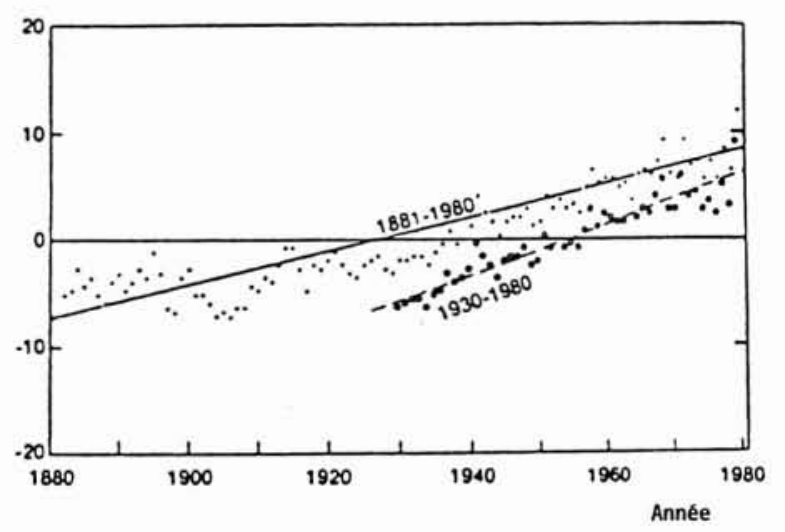

24. Evolution du niveau moyen de la mer de 1880 à 1980 . On note de 1930 à 1980 une croissance légèrement plus accusée mais les ordres de grandeur sont inchangés. marée, du vent, des courants et des variations de pression atmosphérique (puisque l'océan réagit comme un "baromètre inversé " à de telles fluctuations). Un réseau de surveillance à long terme, par altimétrie satellitaire couplée à des stations marines de référence, se met actuellement en place, visant à donner une topographie à grande échelle de la surface de l'océan avec une précision de l'ordre $\mathrm{du} \mathrm{cm}$, ayant le centre de masse de la terre pour point de réfërence géodésique.

- l'extrapolation au futur de l'évolution du niveau marin en fonction de scénarios de réchauffement dûs à un accroissement des concentrations atmosphériques de gaz à effet de serre - équivalent par exemple à un doublement de la concentration actuelle du $\mathrm{CO}_{2}$ atmosphérique - : la question est de savoir si un tel réchauffement se traduira par une fonte (du fait de l'augmentation plus importante de la température aux hautes latitudes) ou un accroissement (du fait de l'accroissement des précipitations dans ces zones) des réserves de glace du continent Antarctique (fig. 25).

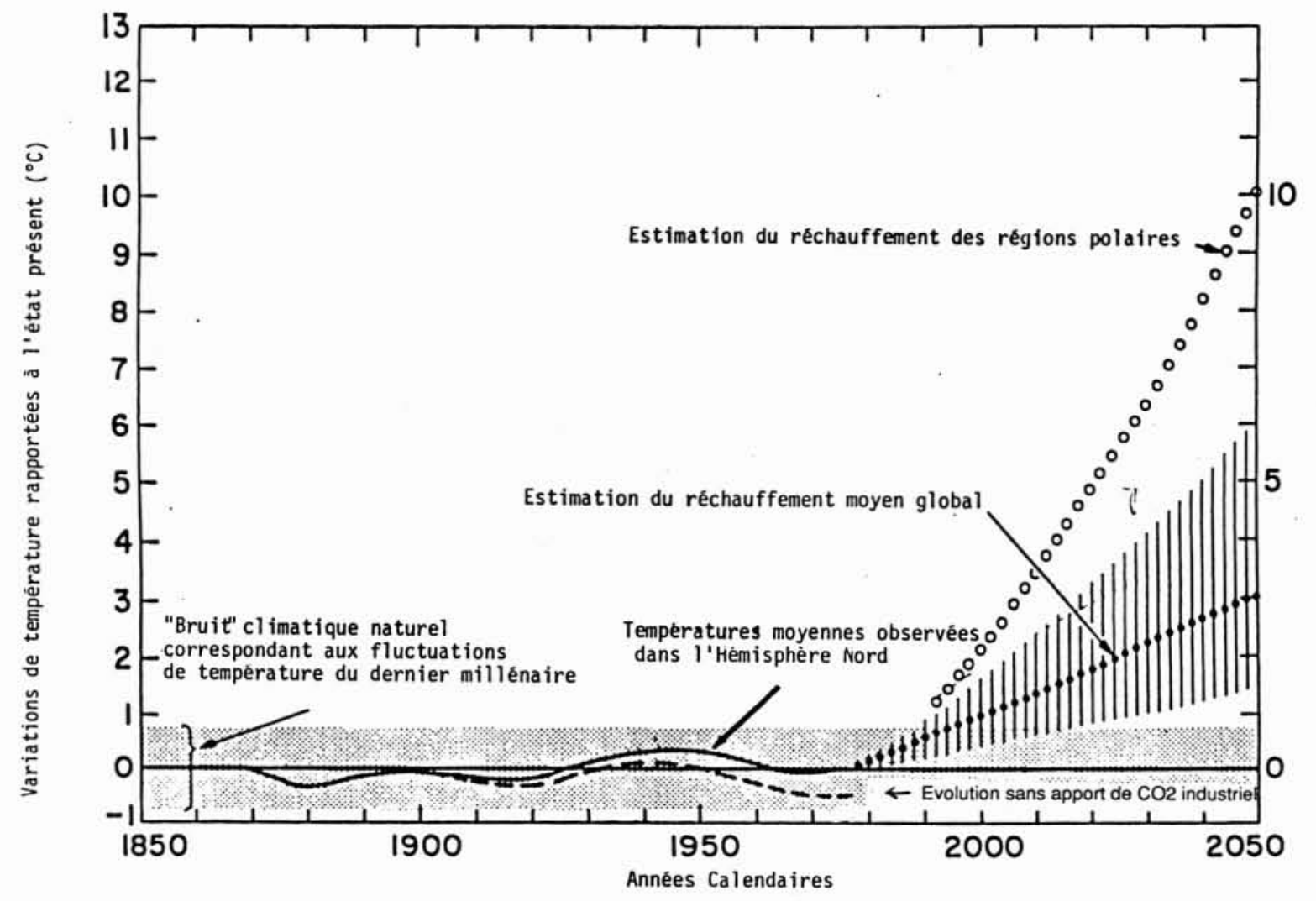

25. Présentation de l'évolution (rapportée à l'état présent) des températures moyennes à la surface de l'Hémisphère Nord de 1850 à 2050.

Le " bruit " climatique naturel d'amplitude $1.5^{\circ} \mathrm{C}$ correspondant aux fluctuations de températures du dernier millénaire (cf. fig. 20) est représenté en grisé. Une simulation de l'évolution des températures de 1900 à 1970 a été faite en supposant aucun apport de $\mathrm{CO}_{2}$ industriel. La projection

dans le futur suppose un doublement de la concentration atmosphérique du $\mathrm{CO}_{2}$ vers 2050 : avec les modèles de simulation utilisés on observerait un réchauffement moyen global de $3{ }^{\circ} \mathrm{C}$, avec $2^{\circ} \mathrm{C}$ en région intertropicale et 8 a $10^{\circ} \mathrm{C}$ aux hautes latitudes (KELLOGG, 1977). Actuellement, ces évaluations sont revues à la baisse avec un échauffement moyen de $1,5^{\circ} \mathrm{C}\left(1{ }^{\circ} \mathrm{C}\right.$ en région intertropicale et 3 a $4{ }^{\circ} \mathrm{C}$ aux hautes latitudes). 


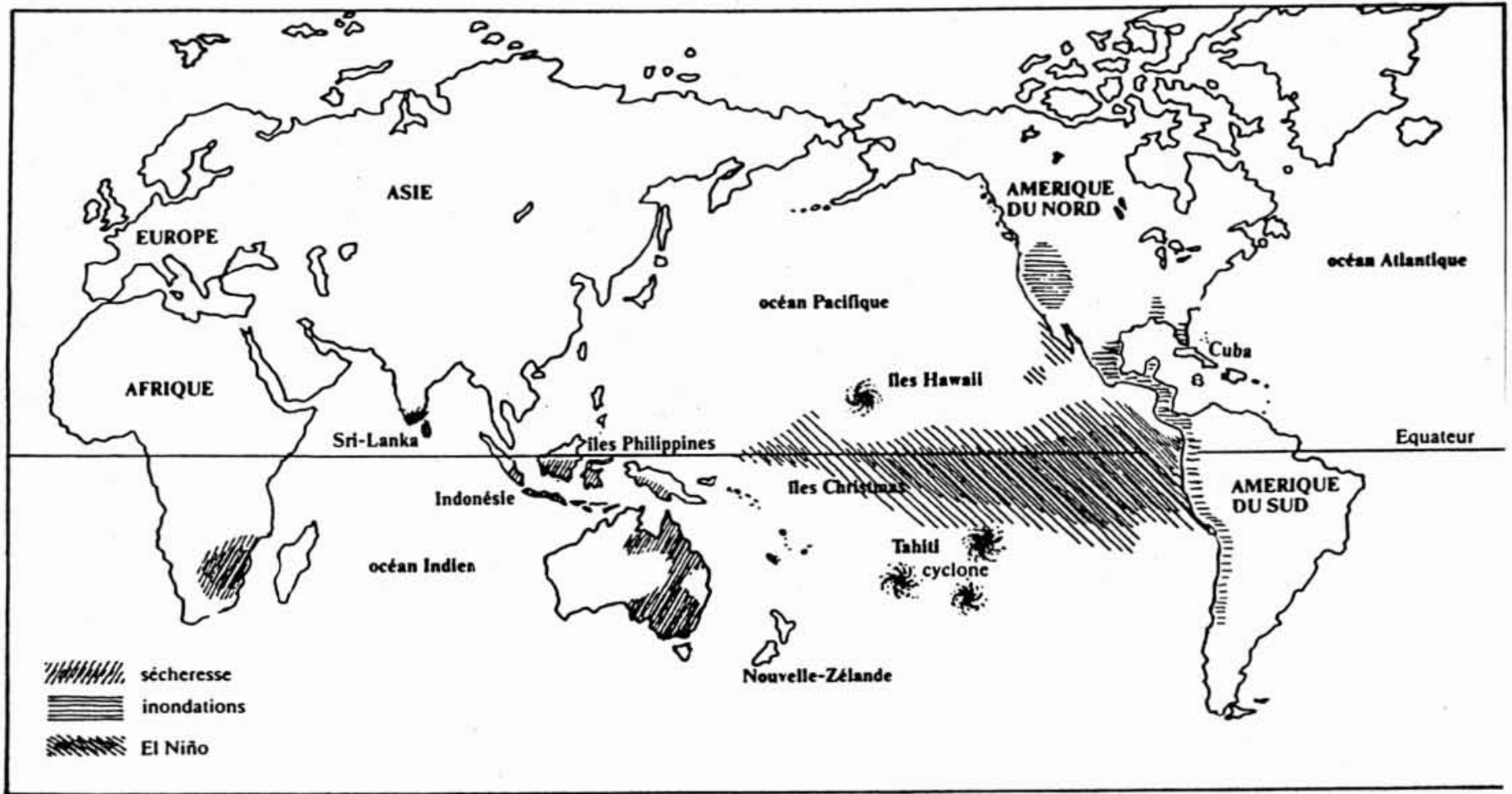

26. L'extension du phénomène El-Niño de 1982-83 dans le Pacifique. L'anomalie de l'extension de la nappe d'eau chaude d'El-Niño a provoqué l'inversion des zones de hautes et basses pressions provoquant sécheresse, inondations et cyclones, qui ont entraîné des dommages chiffrés à 9 milliards de dollars (d'après I. RASOOL et N. SKROTZKY, 1988).

- On dispose maintenant de longues séries chronologiques de paramètres du cycle hydrologique grâce aux réseaux de mesures hydrométéorologiques mis en place depuis un siècle, notamment en ce qui concerne les précipitations et les débits des cours d'eau. A l'échelle du siècle écoulé, la stationnarité statistique de ces séries n'est pas mise en cause et il n'apparaît aucune dérive significative à grande échelle malgré l'importance des fluctuations constatées sur des périodes ou dans des régions plus réduites. Ainsi, bien que très sensible potentiellement aux interventions humaines dans les 3 voies mentionnées plus haut, le cycle hydrologique ne fait encore apparaître aucun signal global des effets de ces interventions à l'échelle du siècle.

- La mise en évidence de l'influence de l'homme sur le climat planétaire est étroitement conditionnée par la fiabilité des techniques d'identification que sont, d'une part, les dispositifs de télédétection appliqués à la surveillance de la composition de l'atmosphère et à l'observation satellitaire de la terre et, d'autre part, la modélisation du comportement dynamique du système géosphère-biosphère soumis à des «forçages » liés aux activités humaines.
Les techniques de mesures satellitaires et de télédétection à partir du sol voient leurs performances et leur fiabilité se développer à un rythme très soutenu : la question à poser ici est celle de leur mise en œuvre pour assurer un suivi continu, homogène en qualité d'observations, sur de longues périodes. La multiplicité des programmes de durée limitée peut être un obstacle à la continuité et à la cohérence des informations recueillies.

La modélisation est loin d'avoir atteint la fiabilité requise pour apporter les réponses attendues. D'abord en ce qui concerne la représentation de la phase atmosphérique des cycles biogéochimiques des composés carbonés, azotés, etc... et des interactions entre les différents compartiments de la biosphère (temps de réponse aux sollicitations, boucles de rétroaction, effets " retard " en relation avec les temps de résidence ou durées de vie de certaines espèces chimiques dans l'atmosphère, etc...). Ensuite, la modélisation de la dynamique climatique présente encore de graves insuffisances dans la prise en compte des couplages océan-atmosphère-cryosphère, du cycle de l'eau, des interactions entre nébulosité et rayonnement, des non- 


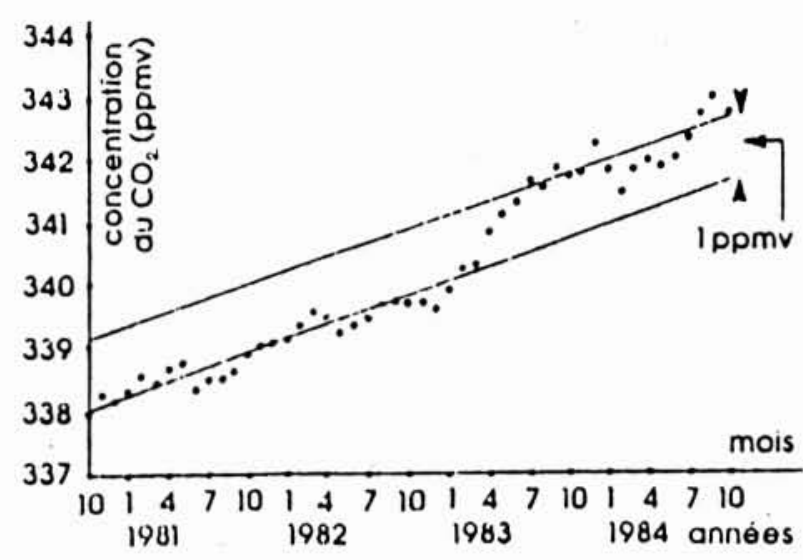

27. Variation des concentrations du $\mathrm{CO}_{2}$ atmosphérique mesurées à l'lle d'Amsterdam dans le Sud de l'Océan Indien, de 1981 a 1984 . On remarque le décalage vers le haut de la courbe de croissance du $\mathrm{CO}_{2}$ durant le phénomène El-Niño de 1982-83: l'amplitude de ce décalage est de 1 ppmv, on retrouve ensuite le même rythme de croissance (d'après A. GAUDRY et al., 1987).

28. Schéma de circulation des cellules de HADLEY dans la zone intertropicale et aux moyennes latitudes. Du fait de l'inclinaison de l'axe de rotation de la terre, la quantité d'énergie du rayonnement solaire reçue par chaque hémisphère varie tous les 6 mois. La zone d'échauffement maximum de la machine terre ne se trouve pas à l'équateur, mais tantôt au tropique du cancer, tantôt au tropique du capricorne. La circulation de l'air qui assure les transferts d'énergie excédentaire sous forme de chaleur latente et de chaleur sensible s'effectue par le jeu des cellules convectives de HADLEY qui se balancent de part et d'autre de l'équateur de façon légèrement asymétrique.
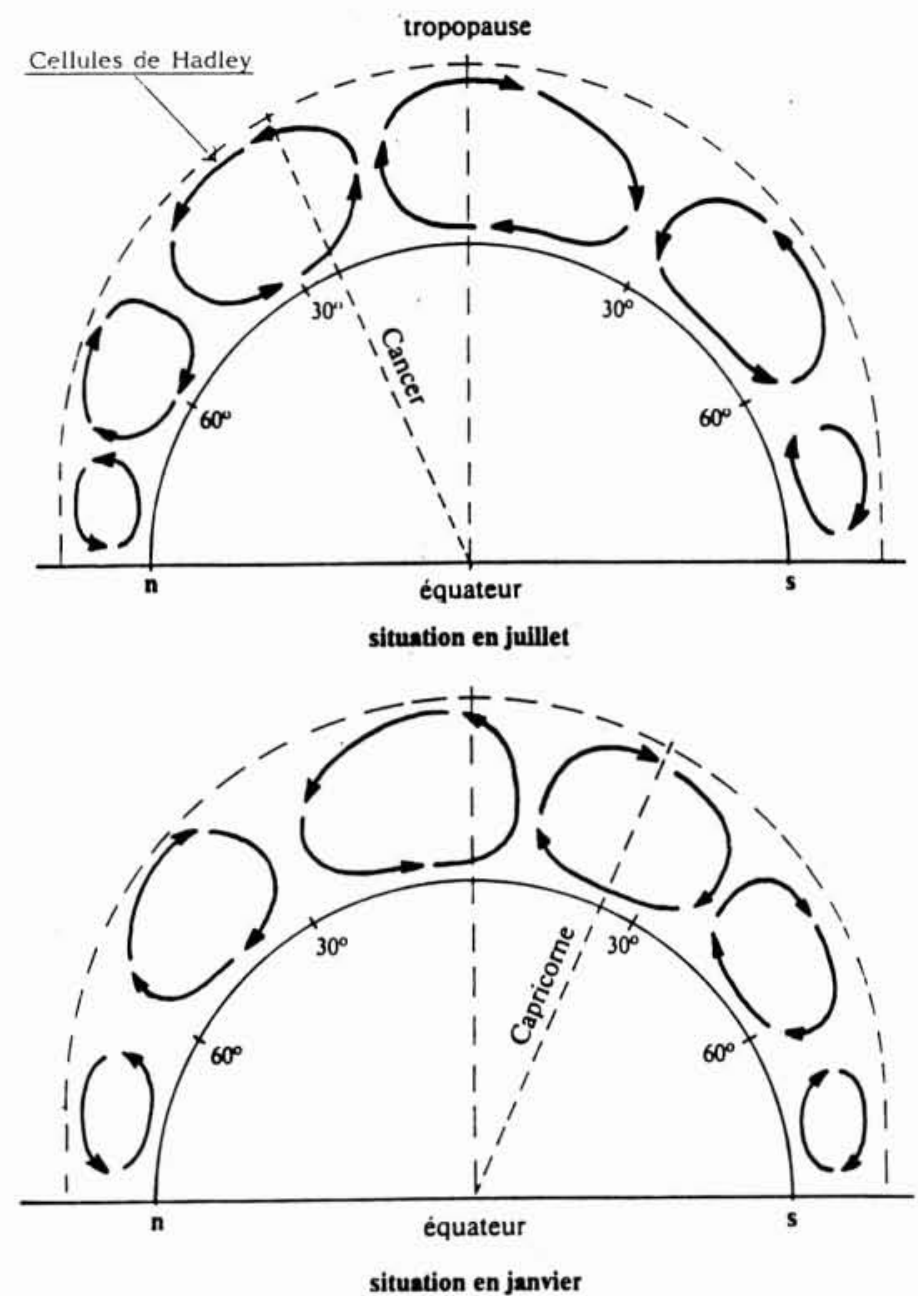

linéarités de réponse du système et des effets antagonistes s'opposant aux tendances au refroidissement ou au réchauffement, etc... Les modèles actuels traitent généralement le problème à l'équilibre et ne permettent pas de fournir des réponses aux phénomènes transitoires : ils sont davantage des modèles de simulation aptes aux études de sensibilité de paramètres que des modèles de prévision adaptés aux identifications d'impact. Un effort de recherche décisif s'impose ici : il est maintenant bien engagé.

\section{Echelle de la décennie : les phénomènes climatiques catastrophiques à grande dimension géographique}

Deux exemples illustreront le caractère extrême de tels phénomènes :

- Le phénomène " El-Niño » est un événement naturel qui se produit chaque année dans la zone équatoriale du Pacifique oriental pendant l'été local de janvier à mars. Les remontées d'eau profonde, riches en éléments nutritifs et favorisant une pêche intensive le long de la côte ouest de l'Amérique du Sud, disparaissent sous une couche d'eau chaude du courant équatorial qui s'écoule vers le Sud. L'intensité du phénomène est variable (en 60 ans on a décompté 8 cas d'El-Niño "renforcés») et peut devenir catastrophique. Ainsi en 1982-83, son extension provoque l'établissement d'une forte dépression au-dessus de ce lac d'eau chaude couvrant une énorme superficie du Pacifique oriental (fig. 26) : il s'ensuivit des précipitations torentielles en Amérique du Nord et du Sud et la formation de cyclones dans le Pacifique Sud. On sait que le phénomène "El-Niño» est lié au déplacement vers le sud-ouest de l'anticyclone du Pacifique Sud: la cause première de ce déplacement est et reste inconnue. Lors de l'été austral 1982-83, ce déplacement atteignit une ampleur inusitée sur plus de $10000 \mathrm{~km}$, transférant ainsi l'anticyclone du Pacifique oriental vers l'Australie et provoquant de ce fait une effroyable sécheresse en Australie et Indonésie, qui se répercuta tout autour de l'Océan Indien avec le glissement vers l'ouest des autres anticyclones austraux. Une conséquence imprévue de l'extension de la nappe d'eau chaude 
en surface du Pacifique intertropical a été un "dégazage " important du $\mathrm{CO}_{2}$ de l'océan, correspondant à l'injection de 6 milliards de tonnes de $\mathrm{CO}_{2}$ dans l'atmosphère, la courbe d'évolution du $\mathrm{CO}_{2}$ atmosphérique se trouvant ainsi décalée de 1 ppmv vers le haut (fig. 27, page précéd.). Trois des facteurs essentiels du climat planétaire - les circulations atmosphérique et océanique, le $\mathrm{CO}_{2}$ atmosphérique - se trouvent être en définitive directement affectés par l'anomalie du phénomène.

- Le second exemple se rapporte à la sécheresse du Sahel, qui a débuté en 1968 et continue à sévir presque sans répit : son explication laisse place à bien des incertitudes. Les pluies de cette région dépendent étroitement des migrations du front intertropical (F.I.T.) où la convergence des alizés provoque le soulèvement des masses d'air humides qui alimentent les cellules de HADLEY, ces migrations pouvant être liées à des variations de température de l'Océan. CHARNEY a proposé en 1974 un mécanisme explicatif possible de l'intensification et de la prolongation de la sécheresse Sahélienne en faisant intervenir une boucle de rétroaction reliant sécheresse et désertification d'après le schéma suivant. Le désert saharien, avec un albedo de 35 à $45 \%$, sec et chaud, émet dans l'infrarouge plus d'énergie qu'il n'en a absorbé du rayonnement solaire, le déficit étant comblé par l'apport de chaleur sensible des masses d'air sec de la branche descendante des cellules de HADLEY (fig. 28, page précéd.). Dans les régions couvertes de végétation, avec un albedo de 10 à $20 \%$, les transferts d'énergie excédentaire de la terre vers l'atmosphère s'opèrent principalement par évaporation, sous forme de chaleur latente permettant de conserver au sol une température modérée et d'alimenter les transferts convectifs d'air humide en altitude. Mais si la végétation disparaît sous l'action de l'homme (surpâturage, etc...), l'albedo augmente et tend à créer un bilan d'énergie déficitaire, limitant les transferts par évaporation et inversant la tendance en renforçant la subsidence des masses d'air sec pour combler le déficit. Un tel processus une fois enclanché, en prolongeant la sécheresse, entraîne la disparition d'encore plus de végétation, et ainsi de suite.

Toutefois cette boucle de rétroaction, reliant l'augmentation de l'albedo sous la pression des activités humaines et l'intensification de la sécheresse, est à elle seule insuf- fisante pour expliquer le phénomène. En effet les variations d'albedo du Sahel, déduites des observations satellitaires, font apparaître un accroissement de 0,20 à 0,30 de 1967 à 1974, puis une diminution de 0,30 à 0,20 de 1974 à 1979 du fait d'une certaine régénération de couverture herbacée et de l'extension des surfaces brûlées. Or, si la grave sécheresse de 1972-73 a bien conduit à une augmentation d'albedo, la diminution de l'albedo constatée en 1979, faisant suite à l'atténuation relative de la sécheresse en 1975-78, n'a pas empêché la réintensification de la sécheresse dans les années 80 .

Il faut donc faire intervenir d'autres facteurs propres à la région ou aux zones voisines.

Le rôle de l'humidité du sol, comparé à celui de l'albedo, a sans doute été sousestimé dans le schéma de CHARNEY car la végétation, en protégeant le sol, peut limiter les pertes par évaporation à celles utiles à la plante. D'autre part le Sahel, caractérisé par une sécheresse qui sévit 9 mois sur 12 , se trouve au nord de la région arrosée par la mousson et l'humidité qu'il reçoit pendant les 3 mois "hors sécheresse" provient en partie du recyclage par évaporation des pluies de mousson tombées plus au Sud. Les dégradations opérées dans les régions soudanoguinéennes par des activités agricoles intensives posent là aussi un problème d'albedo et il faut peut-être rechercher sur ces zones - et pas uniquement dans le Sahel propement dit - dans un affaiblissement de la mousson une des causes de l'entretien de la sécheresse ouest africaine.

Enfin conviendrait-il sans doute de replacer l'accentuation et la persistance du phénomène dans le cadre plus général «d'une fluctuation générale du climat de la terre, qui a commencé à se manifester voici 2 millions d'années environ. La dessication des contrées arides tropicales s'accentue en effet lorsque le volume de glace stocké dans les régions polaires augmente, autrement dit lorsque le climat général du globe tend à devenir plus froid. Or nous sommes actuellement dans une de ces périodes de refroidissement qui a commencé il y a environ 5000 ans... " (J. LABEYRIE).

Réchauffement? Refroidissement? Le débat reste ouvert...

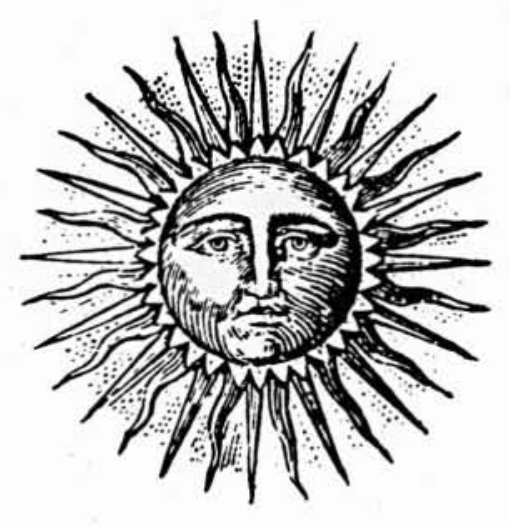




\section{Conclusion : les problèmes du futur}

Au terme de cette description des mouvements climatiques brossée à grands traits, il faut s'interroger sur l'à-venir : - Le futur passe absolument par la compréhension des mécanismes générateurs de ces grands mouvements. Cette compréhension commande à son tour l'évaluation des risques climatiques correspondant à divers scénarios de développement et conduisant à des prises de décision politiques pour prévenir ou limiter ces risques à long terme. Dans l'état actuel des connaissances, les projections faites à l'échelle du siècle à venir pour décrire les conséquences "prévisibles" d'un réchauffement dans le domaine de l'agriculture par exemple, n'engagent que leurs auteurs dans l'impossibilité oủ l'on se trouve de disposer d'instruments fiables de prévision des changements climatiques associés aux scénarios de développement envisagés. Dans le meilleur des cas, il ne peut s'agir que de vraisemblances à soumettre ả la critique. Une donnée "incontournable ", qui relève elle de la certitude, devra être prise en compte dans toutes les études de climatologie prospective : il s'agit de l'explosion démographique en cours dans les pays du Tiers Monde, avec la surexploitation des ressources planétaires qu'elle implique du fait de l'accroissement des besoins associés au développement socio-économique de ces pays dans les domaines de l'énergie et de l'agriculture par exemple. Deux chiffres suffisent à qualifier cette explosion : il aura fallu 2 millions d'années à l'humanité pour atteindre 3 milliards d'individus, 40 ans environ suffiront à doubler ce chiffre...

- Dans ces conditions, un véritable défi climatique est posé à l'aube du siècle à venir : acquérir par la connaissance la maitrise des facteurs du climat pour favoriser une exploitation responsable des ressources planétaires et se protéger des phénomènes extrêmes à conséquences catastrophiques: inondations, typhons et sécheresses. Ces dernières, par leur intensité, leur extension et leur persistance avec les effets cumulatifs qu'elles entraînent, doivent tout particulièrement inciter la communauté scientifique à en rechercher les causes. Une attention permanente au progrès de la connaissance dans ce domaine doit être de règle de la part des décideurs concernés. Il importe que, sur ce problème spécifique, des synthèses scientifiques soient tenues à jour, distinguant les faits établis, les explications recueillant un consensus, les points controversés et les hypothèses relevant de la spéculation théorique.

- Une chance se présente pour mobiliser - pendant au moins une décennie - chercheurs et décideurs sur un programme ambitieux et vital, visant le long terme: le Programme international Géosphère-Biosphère qui couvrira la décennie 1990-1999. A vrai dire, il ne s'agit pas d'un nouveau programme mais plutôt d'un nouvel élan donné aux actions de recherches en cours, avec une coordination et des moyens accrus au niveau mondial, pour répondre à l'attente et aux interrogations des décideurs, des techniciens et du grand public face à l'avenir.

Les objectifs assignés à cette action visent :

- la compréhension des processus interactifs qui régissent le système Géosphère-Biosphère ;

- l'explication des changements intervenus au cours de l'histoire de la terre;

- l'exploration des voies par lesquelles ces processus sont ou peuvent être influencés par les activités humaines ;

- l'identification de l'impact de l'homme sur le fonctionement du système.

On cherche ainsi à constituer un corps de connaissances permettant d'évaluer l'évolution du système GéosphèreBiosphère à l'échelle du siècle à venir. Il est évident que le problème du climat, avec l'explication de ses fluctuations aux diverses échelles considérées ici, constituera un domaine d'investigations privilégié dans le cadre d'une telle action qui s’inscrit dans la durée.

Cette chance, il ne faut pas la laisser passer... 


\section{Références bibliographiques}

Nota : De très larges emprunts ont été faits dans cette présentation aux travaux cités en référence et notamment à ceux de J. LABEYRIE.

Actes du Colloque " $\mathrm{CO}_{2}$ Atmosphérique ", Sophia Antipolis, 67-8 décembre 1984

DuPLessy J.C. et al. - Deglacial Warming of the Northeastern Atlantic Ocean : correlation with the paleoclimatic evolution of the European Continent, Paleogeo., Paleoclimato., Paleoeco., 35 (1981), 121-144.

DUPLESSY J.C. et al. - Oxygen and carbon isotopic composition and biogeographic distribution of planctonic foraminifera in the Indian Ocean, Paleogeo., Paleoclimato., Paleoeco., 33 (1981), 9-46.

Gaudry A. et al. - The 1982-1938 El Niño : a 6 Billion ton $\mathrm{CO}_{2}$ release, Tellus, (1987), 39 B, 209-213.

Kandel R. et Courel M.F. - Le Sahel est-il responsable de sa sécheresse? La Recherche, Paris, Vol. 15, n 158 (1984), $1152-1154$.

LABeyrie J. - L'homme et le climat, Denoël, Paris, 1985.

LABEYRIE J. - Le cadre paléoclimatique depuis 140000 ans, l'Anthropologie, Paris, t. 88 (1984), n 1, 19-48.

LABEYRIE J. - La datation par le carbone-14, La Recherche, Paris, 7 (1976), 1036-1045.

LAmbert G. - Le $\mathrm{CO}_{2}$ dans l'Atmosphère, La Recherche, Paris, $\mathrm{n}^{\circ} 189$ (1987), 778-787.

LoRIus C. - Environnement Atmosphérique et climats passés à partir des archives glaciaires, Courrier du CNRS, Paris, suppl. au n 52 (1983), 26-39.

LoRIus C. - L'évolution des climats, les données du passé, Encyclopédie Scientifique de l'Univers "la Terre, les Eaux, l'Atmosphère ", Bureau des Longitudes, Gauthier Villars, Paris, 1984, 321-337.

Nicholson S. - The Sahara and the nile, A.A. Balkema, Rotterdam, 1980.

RASOOL S.I. et SKROTZKY N. - La Terre, une planète pas comme les autres, Denoël, Paris, 1988.

Rasool S.I. - On Dynamics of Desert and Climate, Colloque M.U.R.S. "L'eau des Hommes en 2000 ». Paris, avril 1987. 